\title{
Fuel and chemistry effects in high Karlovitz premixed turbulent flames
}

\author{
Simon Lapointe ${ }^{a, 1}$ and Guillaume Blanquart ${ }^{b}$ \\ ${ }^{a}$ Graduate Aerospace Laboratories, California Institute of Technology, Pasadena, CA \\ 91125, USA \\ ${ }^{b}$ Mechanical Engineering Department, California Institute of Technology, Pasadena, CA \\ 91125, USA
}

\begin{abstract}
Direct numerical simulations of turbulent premixed flames at high Karlovitz numbers are performed using detailed chemistry. Different fuels, chemical mechanisms, and equivalence ratios are considered and their effects on turbulent flame speed, geometry of the reaction zone, and fuel burning rate are analyzed. Differential diffusion effects are systematically isolated by performing simulations with both non-unity and unity Lewis numbers. Heavy fuels with above unity Lewis numbers are considered. In the unity Lewis number limit, the $n$-heptane, iso-octane, toluene, and methane flames at a given reaction zone Karlovitz number present similar normalized turbulent flame speeds and fuel burning rates close to their respective laminar values. When differential diffusion effects are included, the turbulent flame speeds are lower than their unity Lewis number counterparts due to a reduction in the fuel burning rate. The turbulent reaction zone surface areas increase with the turbulence intensity but aren't strongly affected by fuel, equivalence ratio, chemical mechanism, or differential diffusion. The geometry of the reaction zone is studied through the probability density functions of strain rate and curvature which are very similar when normalized by
\end{abstract}


Kolmogorov scales at the reaction zone. The dependence of the chemical source terms on the scalar dissipation rate in the unity Lewis number case is shown and the distributions of scalar dissipation rate on the reaction surface are similar to those of passive scalars in homogeneous isotropic turbulence. The reduced burning rates in the presence of differential diffusion are discussed. The present results indicate that mean turbulent flame properties such as burning velocity and fuel consumption can be predicted with the knowledge of only a few global laminar flame properties. Once normalized by the corresponding laminar flame quantities, fuel and chemistry effects in high Karlovitz number premixed turbulent flames are mostly limited to differential diffusion.

Key words: turbulent premixed flames, high Karlovitz, differential diffusion effects, flame speed

\section{Introduction}

In the context of Peters' regime diagram $[1,2]$, the interaction between a premixed flame and turbulence is characterized by a velocity ratio $u^{\prime} / S_{L}$ and a length ratio $l / l_{F}$, where $u^{\prime}$ is the turbulence fluctuations, $l$ is the integral length scale, $S_{L}$ is the unstretched laminar flame speed, and $l_{F}$ is the laminar flame thickness $l_{F}=\left(T_{b}-\right.$ $\left.T_{u}\right) / \max (|\nabla T|)$. The relevant dimensionless groups are the Reynolds, Karlovitz, and Damköhler numbers, defined in the present study as

$$
\operatorname{Re}_{l}=\frac{u^{\prime} l}{\nu}, \mathrm{Ka}=\frac{l_{F}}{S_{L}} \sqrt{\frac{u^{\prime 3}}{\nu l}}, \mathrm{Da}=\frac{l / u^{\prime}}{l_{F} / S_{L}}
$$

where $\nu$ is the kinematic viscosity. Only two of these dimensionless numbers are independent since $\mathrm{Ka}^{2} \mathrm{Da}^{2}=\mathrm{Re}_{l}$. Practical premixed combustion devices operate

1 Corresponding author Email address: slapoint@cal tech. edu (S. Lapointe) 
at elevated unburnt temperatures and pressures, under high turbulence intensities, and use heavy hydrocarbon-based fuels. These applications fall in the thin or broken/distributed reaction zones regimes, which are characterized by a large Karlovitz number [3].

High Karlovitz number flames are fundamentally different from low Karlovitz number flames because the turbulence penetrates inside and disturbs the reaction zone. Experiments are difficult to conduct under these conditions and only a limited number of them are available in the literature [4-8]. That is why many studies of turbulent premixed flames in the thin and broken/distributed reaction zones regimes have relied on direct numerical simulations (DNS) with detailed chemistry [9-18]. From these DNS, many insightful observations were made about fluctuations of the chemical source terms, local extinction events, and persistent differential diffusion effects. It was shown that, at high Karlovitz numbers, correlations of chemical source term fluctuations with strain and curvature differed from what is observed in the flamelet regimes [17]. In an effort to reduce the computational cost, simulations have also been performed with simplified chemistry and, in several cases, one-step chemical models were used [19-21]. It is unclear if the choice of chemical model affects the flame-turbulence interactions and what level of details a chemical model should have to capture all relevant physics.

Previous numerical simulations at high Karlovitz numbers using detailed chemistry considered mostly simple fuels such as hydrogen [11-13, 22], methane [14, 15, 22], and propane [14]. Since most transportation fuels are composed of alkane and aromatic species, there is a need to assess the effect of turbulence on the flame for linear alkane, cyclic alkane, and aromatic fuels. Additionally, typical turbulent combustion regime diagrams [1] use only a few laminar flame properties such as $S_{L}$ and $l_{F}$, implicitly assuming no significant chemical effects beyond those global proper- 
ties. This raises an important question: what is the impact of fuel and chemistry on the turbulence-flame interaction?

The present study builds upon previous studies by Savard et al. $[16,17]$ and Lapointe et al. [18] and aims at investigating the role of fuel chemistry in premixed flames at the transition from the thin to broken/distributed reaction zone regimes with engine-relevant, heavy hydrocarbon fuels. In the present work, $n$-heptane/air, toluene/air, iso-octane/air, and methane/air turbulent flames are studied. $n$-heptane, toluene, and iso-octane are characterized by large fuel Lewis numbers $\left(\operatorname{Le}_{F} \approx\right.$ $2.8,2.5$, and 2.8 , respectively) while methane has a close to unity Lewis number $\left(\operatorname{Le}_{F} \approx 1\right)$. Only fuels with Lewis numbers above unity are considered under lean and slightly rich conditions. All the present flames are thermo-diffusively stable. Additionally, different chemical mechanisms of various sizes, from 35 to 207 species, are compared. The focus is placed on the effect of turbulence and differential diffusion on the turbulent flame speeds, the geometry of the reaction zone, and chemical source terms. The objective of the present study is not to compare high Karlovitz flames to low Karlovitz flames, but rather to compare the effects of fuel and chemistry within high Karlovitz number flames.

In Section 2, the computational methodology is reviewed. An overview of the results is presented in Section 3. Section 4 analyzes the effects of turbulence, differential diffusion, and fuel chemistry on the strain rate and curvature distributions at the reaction zone surface. Section 5 compares the chemical fuel consumption rates between the different cases. Finally, the results are summarized in Section 7. 


\section{Computational methodology}

The direct numerical simulations performed in this study follow the methodology presented in Savard et al. [16], Savard and Blanquart [17] and Lapointe et al. [18]. For completeness, the numerical framework is briefly reviewed here. A total of twenty four simulations are used. The parameters for each simulation are provided in Tables 1, 2, 3, and 4. Table 1 lists simulations from previous studies [16, 18] while Tables 2 to 4 list new simulations performed in the context of the present study.

\subsection{Flow configuration}

The same flow configuration is used for all the simulations and is illustrated in Fig. 1. It was chosen to achieve a statistically-planar, freely propagating flame without mean shear and mean curvature effects. The simulations can be run for an unbounded time, allowing to reach a statistically-stationary state. Different configurations, where turbulence is sustained through mean shear in the flow, have been used in DNS studies of premixed turbulent combustion [23-25]. However, these configurations require directing computational resources towards large domains to contain the mean shear. Additionally, these flows develop in space or time which increases the complexity of isolating the effects of the flame on turbulence. Therefore, the present configuration was chosen in order to study the effect of turbulence on the chemical source terms at a reasonable computational cost.

Since the flow configuration lacks generation of turbulence due to large scale flow straining, turbulence forcing is necessary [9-12, 14, 19-21]. The forcing magnitude is increased $0.5 L$ (where $L$ is the domain width) downstream of the inlet to 
reach the desired TKE value. The velocity field forcing is stopped after a distance of $8 L$ and the velocity fluctuations decay rapidly, such that there are no negative axial velocities at the outlet. The forcing method used is briefly described in subsection 2.3. For each simulation, the mean inlet velocity is constant throughout the simulation and selected to match approximately the turbulent flame speed of that simulation. In practice, the flames drift slightly, but the drift is negligible compared to the domain length. The simulations are performed for at least 50 eddy turnover times, $\tau=k / \epsilon$, and statistics are computed using the last $30 \tau$ to remove any initial transient effects. It should be noted that a turnover time is shorter than a flame time $\left(\tau_{F}=l_{F} / S_{L}\right)$ at high Ka. Simulations were thus performed for $\sim 5 \tau_{F}$ for case A to $\sim \tau_{F}$ for case D. Using $30 \tau$ was verified to be sufficient by running one of the simulations for approximately ten flame times. The authors have found that only a few eddy turnover times were sufficient to remove the effect of the initial conditions on quantities controlled by small turbulent scales such as chemical source terms, curvature, and strain rate. All simulations are performed on uniform grids with constant $\Delta x$ spacing and offer sufficient resolution of the small-scale turbulence $\left(\Delta x \leq 2 \eta_{u}\right)[26]$ and sufficient resolution of the flame $\left(\Delta x \leq l_{F} / 20\right)$. Simulations were performed with a timestep size restricted by the convective CFL; $\Delta t<\Delta x /|u|$. This leads to timesteps ranging from $\Delta t \approx 1 \times 10^{-6}$ s for case A to $\Delta t \approx 1 \times 10^{-8} \mathrm{~s}$ for case $\mathrm{D}$. Grid resolution was assessed by Lapointe et al. [18] and the time accuracy (timestep size and associated errors) was also previously investigated [27, 28], both were found to be sufficient.

[Fig. 1 about here.]

The objective of the present study is to investigate the effects of small-scale turbulence on the flame chemistry at high Karlovitz numbers. Given that purpose and computational cost considerations, a small integral length scale (roughly equal to 
the laminar flame thickness) is used. It is important to note that the simulations may be missing large scale effects. However, the focus of the paper is on the reaction zone, whose thickness is significantly smaller than the domain size $\left(L \sim 15 \delta_{F, \text { lam }}\right)$. This way, the effects of small-scale turbulence on the flame can be isolated form large-scale effects (not specific to high Karlovitz number flames and already present in low Karlovitz number flames).

\subsection{Governing equations}

The low Mach number Navier-Stokes equations are solved

$$
\begin{gathered}
\frac{\partial \rho}{\partial t}+\nabla \cdot(\rho \mathbf{u})=0 \\
\frac{\partial \rho \mathbf{u}}{\partial t}+\nabla \cdot(\rho \mathbf{u u})=-\nabla p+\nabla \cdot \tau+\mathbf{f} \\
\frac{\partial \rho Y_{i}}{\partial t}+\nabla \cdot\left(\rho \mathbf{u} Y_{i}\right)=-\nabla \cdot \mathbf{j}_{\mathbf{i}}+\rho \dot{\omega}_{i} \\
\frac{\partial \rho T}{\partial t}+\nabla \cdot(\rho \mathbf{u} T)=\nabla \cdot(\rho \alpha \nabla T)+\rho \dot{\omega}_{T} \\
-\frac{1}{c_{p}} \sum_{i} c_{p, i} \mathbf{j}_{\mathbf{i}} \cdot \nabla T+\frac{\rho \alpha}{c_{p}} \nabla c_{p} \cdot \nabla T
\end{gathered}
$$

In these equations, $\mathbf{u}$ is the velocity vector, $\rho$ is the fluid density, $\tau$ is the viscous stress tensor, $\mathbf{f}$ is the turbulence forcing term, $Y_{i}$ is the species mass fraction, $\dot{\omega}_{i}$ is the species production rate, $\mathbf{j}_{\mathbf{i}}$ is the species diffusion mass flux, $T$ is the temperature, $c_{p, i}$ is the species heat capacity, $c_{p}$ is the mixture heat capacity, $\alpha=\lambda /\left(\rho c_{p}\right)$ is the mixture thermal diffusivity, $\lambda$ is the mixture thermal conductivity, and $\dot{\omega}_{T}=-\frac{1}{c_{p}} \sum h_{i} \dot{\omega}_{i}$ is the temperature production term (heat release). The 
species diffusion term is defined as:

$$
\mathbf{j}_{\mathbf{i}}=-\rho D_{i} \frac{Y_{i}}{X_{i}} \nabla X_{i}-\rho Y_{i} \mathbf{u}_{\mathbf{c}}
$$

where $\mathbf{u}_{\mathbf{c}}$ is the correction velocity to ensure conservation of mass:

$$
\mathbf{u}_{\mathbf{c}}=-\sum_{i} \rho D_{i} \frac{Y_{i}}{X_{i}} \nabla X_{i}
$$

The fluid is treated as a perfect gas with the equation of state

$$
P_{0}=\rho R T \sum_{i} \frac{Y_{i}}{W_{i}}
$$

where $P_{0}$ is the thermodynamic pressure, $R$ is the universal gas constant, and $W_{i}$ is the species molecular weight.

The governing equations 2 to 8 are solved using the energy conservative, finite difference code NGA designed for the simulation of variable density, low Mach number, turbulent flows [29]. The simulations use second-order accurate spatial and temporal discretizations. A semi-implicit Crank-Nicolson time integration is used [30]. A preconditioning strategy based on a diagonal approximation of the chemical Jacobian [28] is used to integrate the chemical source terms. This integration technique is a critical element of the present numerical simulations as it leads to a computational cost linear with the number of species. The third-order Bounded QUICK scheme, BQUICK [31], is used as the transport scheme of the species and temperature to ensure that the scalars remain within their physical bounds. 


\subsection{Turbulence forcing}

The canonical flow configuration used in the present study lacks generation of turbulence due to large scale flow straining. Consequently, the turbulence is expected to decay. Velocity field forcing is thus necessary to maintain a constant turbulence intensity and achieve a statistically-stationary state.

Linear velocity forcing [32-34] was chosen for its physical nature and good stability properties. The linear forcing method mimics the missing large scale straining through the addition of a source term to the momentum equation

$$
\mathbf{f}(x, y, z, t)=\frac{\epsilon_{0}}{2 k(x, t)}(\rho \mathbf{u}(x, y, z, t)-\overline{\rho \mathbf{u}}(x, t)),
$$

where $\epsilon_{0}$ is the desired dissipation rate and $k$ is the planar Favre-averaged TKE

$$
k=\frac{1}{2}\left(\widetilde{u^{\prime \prime 2}}+\widetilde{v^{\prime \prime 2}}+\widetilde{w^{\prime \prime 2}}\right) .
$$

Planar Favre averages are defined as

$$
\widetilde{\xi}=\frac{\overline{\rho \xi}}{\bar{\rho}}
$$

with the standard (Reynolds) planar average

$$
\bar{\xi}(x, t)=\frac{1}{L^{2}} \int_{0}^{L} \int_{0}^{L} \xi(x, y, z, t) \mathrm{d} y \mathrm{~d} z
$$

Energy and dissipation spectra obtained with the present implementation for cases $\mathrm{A}_{\mathrm{NC} 7}$ through $\mathrm{D}_{\mathrm{NC} 7}$ were presented by Lapointe et al. [18]. They show that the small scale turbulent structures altering the flame structure and the reaction zone follow 
the expected universal behavior [26] despite the relatively low Reynolds number. Additionally, in recent work [35], the present configuration with forced turbulence was found to produce similar vorticity characteristics as the slot Bunsen flames of Sankaran et al. [23].

\subsection{Simulation parameters}

For each simulation, the unburnt gas is a fuel/air mixture at standard pressure $\left(P_{0}=1 \mathrm{~atm}\right)$ and varying temperatures. The unburnt temperature $\left(T_{u}\right)$ is changed to cover a range of reaction zone Karlovitz numbers clustered around the thin to broken/distributed reaction zones regimes. Table 1 summarizes the simulation parameters of the $n$-heptane simulations. For each case, two simulations were performed: one with non-unity Lewis numbers and one with unity Lewis numbers (subscript 1). Simulations with unity Lewis numbers are not expected to reproduce realistic, experimentally observed flames. They are used for comparison purposes as they allow to systematically isolate the effects of differential diffusion from those of turbulence. Simulations $\mathrm{A}_{\mathrm{NC} 7}$ through $\mathrm{D}_{\mathrm{NC} 7}$ were previously reported by Lapointe et al. [18]. In the present work, cases B and C are simulated again with different fuels, equivalence ratios, and chemical mechanisms. Figure 2 shows where the simulations stand on a typical regime diagram.

[Fig. 2 about here.]

First, the equivalence ratio is varied by performing case $\mathrm{B}$ at $\phi=0.7$ and $\phi=$ 1.3 (cases $\mathrm{B}_{\mathrm{NC} 7, \phi=0.7}$ and $\mathrm{B}_{\mathrm{NC} 7, \phi=1.3}$ ) (see Table 2). Compared to the baseline case $(\phi=0.9)$, leaner conditions $(\phi=0.7)$ are selected to determine if differential diffusion is enhanced, and richer conditions $(\phi=1.3)$ are chosen to determine if 
the fuel Lewis number $\mathrm{Le}_{F}$ is still a controlling variable even if the fuel is not the limiting reactant. Case B is chosen since it presents strong differential diffusion effects, namely a significant reduction in the chemical source terms compared to the corresponding one-dimensional laminar flame $[17,18]$.

Four different fuels are considered: $n$-heptane (abbreviated NC7), iso-octane (abbreviated iC8), toluene (abbreviated ACH3), and methane (CH4). Toluene and isooctane are chosen because they have similar fuel Lewis numbers to $n$-heptane but have distinct and complex fuel decomposition chemistry. Methane is chosen for comparison purposes because of its close to unity fuel Lewis number and simpler chemistry. The fuel Lewis numbers used in the non-unity Lewis number simulations are $\mathrm{Le}_{n \mathrm{C} 7}=2.84, \mathrm{Le}_{\mathrm{ACH} 3}=2.45, \mathrm{Le}_{i \mathrm{C} 8}=2.81$, and $\mathrm{Le}_{\mathrm{CH} 4}=0.99$. Simulations $\mathrm{B}_{\mathrm{CH} 4}$ and $\mathrm{B}_{\mathrm{ACH} 3}$ present similar turbulence conditions and laminar flame properties as case $\mathrm{B}$ but use different fuels. Similarly, simulations $\mathrm{C}_{\mathrm{ACH} 3}$ and $\mathrm{C}_{\mathrm{iC} 8}$ are performed at the same turbulent conditions as simulations $\mathrm{C}$ and $\mathrm{C}_{1}$ to investigate fuel effects (see Table 3).

Finally, simulations $\mathrm{B}_{\mathrm{NC}, \mathrm{CM}}$ and $\mathrm{C}_{\mathrm{NC} 7, \mathrm{JSF}}$ present similar conditions as cases $\mathrm{B}$ and C but use larger, more detailed chemical mechanisms (see Table 4). Cases B and $\mathrm{C}$ are chosen to compare fuel and chemical models since they use different unburnt temperatures, present significant differential diffusion effects, and are at the transition between thin and distributed reaction zones.

[Table 1 about here.]

[Table 2 about here.]

[Table 3 about here.]

[Table 4 about here.] 


\subsection{Chemical and transport models}

As mentioned earlier, simulations with four different fuels are performed. Methane/air simulations use the GRI 3.0 mechanism [36] without nitrogen chemistry with 36 species and 420 reactions (forward and backward reactions are counted separately). Cases $\mathrm{B}_{\mathrm{NC} 7, \mathrm{CM}}$ use the full CaltechMech (172 species, 1853 reactions) [37]. Cases $\mathrm{C}_{\mathrm{NC} \text {,JSF }}$ use the JetSurF 2.0 mechanism [38] (207 species, 2477 reactions). Isooctane/air simulations use CaltechMech without soot and linear alkane decomposition pathways (74 species, 976 reactions). Toluene/air simulations use the 47 species and 290 reactions mechanism developed in Bisetti et al. [39]. The baseline $n$-heptane simulations use a further reduced version of that mechanism consisting of 35 species and 217 reactions. Species produced under rich conditions (mostly aromatic species) were removed.

Given the size of the chemical models used (up to 207 species), several simplifications are made in evaluating mixture-averaged transport coefficients. The species viscosities $\mu_{i}$ are computed from standard gas kinetic theory [40] and the mixtureaveraged viscosity is calculated using a modified form of Wilke's formula [18, 41]. The species thermal conductivities, $\lambda_{i}$, are computed using Eucken's formula [42] and the mixture-averaged thermal conductivity $\lambda$ is obtained following Mathur et al. [43]. The species diffusivities are computed as $D_{i}=\alpha / \mathrm{Le}_{i}$, with the species Lewis numbers $\mathrm{Le}_{i}$ assumed to be constant throughout the flame. The species Lewis numbers are taken from simulations of one-dimensional, laminar, premixed flames using a mixture-averaged formulation for transport properties computed with FlameMaster [44]. It was shown by Savard [27] and Burali et al. [45] that, in the turbulent heptane flame considered (case $\mathrm{B}_{\mathrm{NC} 7}$ ), differences in species mass fractions and chemical source terms were negligible between mixture-averaged for- 
mulation for transport properties and constant Lewis numbers. Soret and Dufour effects are neglected. The impact of neglecting Soret and Dufour effects in 3D turbulent flames is still unknown and is beyond the scope of the present work. Using these simplifications, the computational time required to evaluate transport properties scales linearly with the number of species. This allows the use of large, detailed chemical models at reasonable computational costs.

\section{Turbulent flame speed}

The reader is referred to the recent work of Lapointe et al. [18] for a qualitative overview of the $n$-heptane simulations including instantaneous vorticity. At a given reaction zone Karlovitz number (similar turbulent conditions), the instantaneous fields from the present simulations with different fuels, equivalence ratios, and mechanisms are qualitatively (visually) similar to the previous $n$-heptane simulations performed. Instantaneous temperature and fuel consumption rate contours for all cases used in this study are provided as supplemental material. In this section, the effects of fuel, chemistry, and transport effects on the turbulent flame speeds are quantified.

\subsection{Evaluating the mean turbulent flame speed}

The mean turbulent flame speed may be evaluated in different ways from the previous simulation results. The instantaneous turbulent flame speed is defined here as the volume integral of the fuel consumption rate:

$$
S_{T}=\frac{1}{\left(\rho Y_{F}\right)_{u} L^{2}} \int_{V}-\rho \dot{\omega}_{F} \mathrm{~d} V
$$


The instantaneous flame speed is computed for each snapshot (data file) saved (approximately one eddy turnover time apart) and then averaged (over time) to obtain the mean turbulent flame speed $\overline{S_{T}}$. As the differences between the various fuels, equivalence ratios, and chemical mechanisms may be small, it is important to ensure that the statistical error on the quantities of interest is small. In order to reduce the error on $\overline{S_{T}}$ and other mean quantities, data is collected over 30 eddy turnover times. This allows to capture multiple cycles of the lower frequency phenomena and provides a sufficient number of snapshots to constitute a representative sample.

As mentioned in Section 2.1, the inlet bulk velocity black is constant and differs slightly from the turbulent flame speed. Therefore, the flame drifts from its initial position. In all cases, the drift is negligible compared to the domain size. The flame drift can be computed as in Savard and Blanquart [17]

$$
\Delta x_{f}=x_{f}-x_{0},
$$

where $x_{0}$ is the position of the flame at time $t=0$ and $x_{f}$ is the instantaneous position of the flame, computed as

$$
x_{f}=11 L-\frac{1}{L^{2} c_{b}} \int_{V} c \mathrm{~d} V
$$

where $c=Y_{\mathrm{H}_{2}}+Y_{\mathrm{H}_{2} \mathrm{O}}+Y_{\mathrm{CO}}+Y_{\mathrm{CO}_{2}}$ is used as a progress variable [46] and takes the value $c_{b}$ in the burnt gas. Then, the mean turbulent flame speed can be evaluated from the change in flame position as

$$
\bar{S}_{\text {drift }}=\frac{\Delta x_{f}}{\Delta t}+U_{\text {inlet }} .
$$

Figure 3 shows the instantaneous flame speed plotted against time for case $\mathrm{B}_{n \mathrm{C} 7, \phi 1.3,1}$. 
Large fluctuations are the mean value are observed. As will be shown later (Section 3.3), these fluctuations are due to changes in the topology of the reaction zone surface. The mean and standard deviations of the turbulent flame speeds computed from the instantaneous flame speed (using Eq. 13) at every timestep (black line) and the instantaneous flame speed from the snapshots used for the statistics (red dots) are virtually the same $(0.75 \pm 0.20 \mathrm{~m} / \mathrm{s}$ and $0.76 \pm 0.21 \mathrm{~m} / \mathrm{s}$, respectively). The agreement suggests that the mean flame speeds computed from a limited number of data files are accurate estimates over the data collection period. Additionally, the mean turbulent flame speed computed from the flame drift (Eq. 16) over the same period is $0.72 \mathrm{~m} / \mathrm{s}$, which confirms that both methods yield very similar mean flame speeds.

[Fig. 3 about here.]

\subsection{Chemistry and transport effects}

Figure 4 presents the mean turbulent flame speeds normalized by the corresponding unstretched laminar flame speeds. Filled symbols correspond to the non-unity Lewis number cases while empty symbols correspond to the unity Lewis number cases. The dashed lines represent power law fits of the form $\overline{S_{T}} / S_{L}=1+a \mathrm{Ka}_{\delta}^{b}$.

Since simulations at different unburnt temperatures are used, the flame speeds are plotted against the reaction zone Karlovitz number:

$$
\mathrm{Ka}_{\delta}=\frac{\delta_{F, \mathrm{lam}}^{2}}{\eta_{\delta}^{2}}
$$

where $\delta_{F, \text { lam }}$ is the laminar reaction zone thickness and $\eta_{\delta}$ denotes the value of the Kolmogorov length scale at the reaction zone (taken at $T_{\text {peak }}$, the temperature 
corresponding to the peak value of the fuel consumption rate in the flame). The laminar reaction zone thickness is defined here as the distance over which the fuel burning rate is greater than $5 \%$ of the maximum laminar value. For the flames considered here, $\delta_{F}$ varies from $1 / 3$ to $1 / 2$ of the laminar flame thickness $\left(l_{F}\right)$. An increase in the unburnt temperature leads to a reduction in the viscosity ratio across the flame. As the Kolmogorov length scale depends on the viscosity, small-scale turbulent structures penetrate further in the reaction zone at higher unburnt temperatures. Considering a fixed velocity ratio $u^{\prime} / S_{L}$ and length ratio $l / l_{F}$, the change in viscosity ratio leads to an increased Karlovitz number at the reaction zone while the Karlovitz number in the unburnt gases does not change. To take these effects into account, Lapointe et al. [18] showed it is more adequate to use the reaction zone Karlovitz number $[1,2]$ when comparing reaction rates. It is important to note that the turbulent flame speed is expected to depend also on the ratio of integral length scale to flame thickness. This ratio was intentionally kept around unity for all flames.

[Fig. 4 about here.]

As expected, the turbulent flame speed increases with $\mathrm{Ka}_{\delta}$ [1]. There is also a clear difference between non-unity Lewis number simulations and unity Lewis number simulations. The ratio of turbulent to laminar flame speed is always lower when differential diffusion is included although both flames are subjected to the same incoming turbulent flow. This is typical of heavy fuels with above unity Lewis numbers under thermo-diffusively stable conditions. More specifically for the present objectives, very similar mean turbulent flame speeds are obtained with the different equivalence ratios, fuels, and chemical mechanisms. This suggests that equivalence ratios, fuels, and chemical mechanisms only have limited effects on the present turbulent flames. 


\subsection{Global vs. local effects}

Dating back to Damköhler [47], turbulence-flame interactions have been described conceptually by considering two limiting regimes of large-scale and small-scale turbulence. In the large-scale regime (corrugated flamelet regime), Damköhler proposed that the turbulent flame speed is proportional to the turbulent flame surface area $A_{T}$, or $S_{T} / S_{L} \approx A_{T} / A$ where $A$ is the cross-section area $\left(A=L^{2}\right)$. In contrast, for a flame interacting with small-scale turbulence (thin reaction zone regime) as for all present flames, Damköhler proposed that the turbulent flame speed should be proportional to the turbulent diffusivity, $S_{T} \sim \sqrt{D_{T} / \tau_{c}}$ where $D_{T}$ is the turbulent diffusivity and $\tau_{c}$ is the chemical time scale. Substituting the chemical time scale by the inverse of the fuel burning rate $\left(\dot{\omega}_{F}\right)$ would yield the following expression:

$$
\frac{S_{T}}{S_{L}} \approx \sqrt{\frac{D+D_{T}}{D} \frac{\left\langle\dot{\omega}_{F} \mid T_{\text {peak }}\right\rangle}{\dot{\omega}_{F, \text { lam }}}},
$$

where $D$ is the laminar diffusivity in the unburnt gases.

However, this expression has not yet been verified in simulations of high Karlovitz number premixed flames. For instance, it was shown by Poludnenko and Oran [20], for a turbulent flame close to the transition between the thin reaction zone and distributed burning regimes, that a strong correlation could be found between the turbulent flame speed and the surface area at the reaction zone. Additionally, as was discussed in Savard and Blanquart [17], even if the present flames are not in the corrugated flamelet regime, the reaction zones remain thin and are only weakly corrugated.

Following these observations, a similar approach to that used by Damköhler for the corrugated flamelet regime is taken here considering a reaction zone surface $(T=$ 
$\left.T_{\text {peak }}\right)$. By rewriting Eq. 13 as a surface integral along the isocontour $T=T_{\text {peak }}$ and an integral in the normal direction and using the observation of Savard and Blanquart [17] that the fuel consumption locally scales like its value at $T=T_{\text {peak }}$, the turbulent flame speed can be approximated as:

$$
\frac{S_{T}}{S_{L}} \approx \frac{A_{T}}{A} \frac{\left\langle\dot{\omega}_{F} /|\nabla T| \mid T_{\text {peak }}\right\rangle}{\dot{\omega}_{F, \text { lam }} /\left|\nabla T_{\text {lam }}\right|} .
$$

More details on the derivation can be found in Appendix A. It is important to note that, as opposed to Savard and Blanquart [17], it is not assumed that the temperature gradient at the reaction zone is equal to its laminar counterpart. This equation stresses that the local flame propagation speed is not the same as the laminar flame speed and thus changes need to be made to modeling approaches based on unstretched laminar flames.

Note that Eq. 19 is equivalent to the burning velocity model proposed by Bray [48] and Candel and Poinsot [49]:

$$
\frac{S_{T}}{S_{L}}=\frac{A_{T}}{A} I_{0}
$$

if the burning efficiency factor is replaced by the ratio of the turbulent to laminar burning rate (divided by the local temperature gradient) at the reaction surface. Instantaneous contours of $\dot{\omega}_{F} /|\nabla T|$ for the $n$-heptane cases are included as supplemental material.

The validity of Eq. 19 is first verified by plotting the instantaneous turbulent flame speed, surface area, Eq. 19, and the burning efficiency factor $\left(I_{0}=\frac{S_{T} / S_{L}}{A_{T} / A}\right)$, for cases $\mathrm{C}_{\mathrm{ACH} 3}$ (non-unity and unity Le), as shown in Fig. 5. The turbulent flame surface area, defined as the area of an isotherm, is computed using a marching cubes algorithm [50]. The turbulent flame speed and surface area both exhibit very similar temporal evolutions and are characterized by large fluctuations of the order of 
their mean values. Eq. 19 is very close to $S_{T} / S_{L}$. On the other hand, the burning efficiency factor presents much smaller fluctuations. These observations lead to two main conclusions. First, Eq. 19 holds, or equivalently:

$$
I_{0} \approx \frac{\left\langle\dot{\omega}_{F} /|\nabla T| \mid T_{\text {peak }}\right\rangle}{\dot{\omega}_{F, \text { lam }} /\left|\nabla T_{\text {lam }}\right|}
$$

Second, the large fluctuations in $S_{T}$ are mostly due to $A_{T}$ since the chemical source terms present much smaller fluctuations.

Equation 19 is further tested by plotting the instantaneous $A_{T}\left\langle\dot{\omega}_{F} /|\nabla T| \mid T_{\text {peak }}\right\rangle /\left(A \dot{\omega}_{F, \text { lam }} /\left|\nabla T_{\text {lam }}\right|\right)$ against the instantaneous turbulent flame speed for each snapshot used for the statistics from all simulations. As shown in Fig. 6, most points fall along a straight line, indicating that Eq. 19 holds for the present flames. It is important to note that the instantaneous turbulent flame speed varies by about an order of magnitude across all simulated flames. Equation 19 is expected to hold under thermo-diffusively stable conditions. It is unclear if it holds for thermo-diffusively unstable conditions, such as lean hydrogen/air flames. This is beyond the scope of the present study and should be the subject of future work.

[Fig. 5 about here.]

[Fig. 6 about here.]

Equation 19 distinguishes the local effects of turbulence on the chemistry (represented by $\left.\left\langle\dot{\omega}_{F} /|\nabla T| \mid T_{\text {peak }}\right\rangle\right)$ from the global propagation of the flame into incoming turbulence (represented by $A_{T}$ ). The flame surface area responds to the large turbulent scales while the chemical source terms are mostly affected by small scales (smaller than the reaction zone thickness $\delta_{F}$ ). These two factors are analyzed separately below. 
The ratio of flame surface area to domain cross-section area $\left(A_{T} / A\right)$ is computed at the temperature of peak fuel consumption rate, $T_{\text {peak }}$, for the different cases and plotted against the reaction zone Karlovitz number in Fig. 7a. As mentioned earlier, changes in the flame surface area are investigated here at a fixed $l / l_{F}$. As discussed in previous studies $[1,21]$, these large scales are also expected to influence $A_{T}$. In the present cases, the turbulent flame surface area increases with increasing $\mathrm{Ka}_{\delta}$, suggesting increased small-scale wrinkling. The flame surface area does not change significantly between non-unity and unity Lewis number flames at a fixed $\mathrm{Ka}_{\delta}$. This may not be the case for thermo-diffusively unstable conditions.

[Fig. 7 about here.]

Figure $7 \mathrm{~b}$ presents the conditional means of $\dot{\omega}_{F} /|\nabla T|$ at $T_{\text {peak }}$ as a function of the reaction zone Karlovitz number for the different simulations using both non-unity and unity Lewis numbers. The mean fuel consumption rates of the unity Lewis number cases are close to the laminar values (with deviations less than 10\%). This holds for all fuels used here as well as the different mechanisms and equivalence ratios. On the other hand, in the non-unity Lewis number cases, the mean fuel consumption rate is lower than its unity Lewis number counterpart in all cases (as expected with the present fuels with $\mathrm{Le}_{F}>1$ ). $\mathrm{As} \mathrm{Ka}_{\delta}$ is increased, the conditional means get closer to the corresponding unity Lewis number flame as turbulent mixing takes over molecular mixing. It is important to note that the conditional means of $n$-heptane, toluene, and iso-octane (which have similar fuel Lewis numbers) are very similar. Despite the different chemical pathways of the heavy hydrocarbon fuels considered, there are no large differences in their normalized fuel consumption rates at $T_{\text {peak }}$ at a given $\mathrm{Ka}_{\delta}$. The differences in fuel consumption rates between the different heavy fuels are small but the differences between unity and non-unity Lewis numbers are significant. Unsurprisingly, the methane case, which has a fuel 
Lewis number close to unity, presents a larger conditional mean, closer to the unity Lewis number methane flame. Additionally, the mean fuel consumption rate for case $\mathrm{B}_{n C 7, \phi 1.3}$ is very close to cases $\mathrm{B}_{n C 7}$ and $\mathrm{B}_{n C 7, \phi 0.7}$. The Lewis number of oxygen (the limiting reactant) being close to unity, $\mathrm{Le}_{\mathrm{O}_{2}}=1.06$, it appears that the fuel Lewis number is still the controlling parameter for $\phi>1$.

In summary, together with the mean turbulent to laminar chemical source term $\langle\dot{\omega} /|\nabla T|\rangle /(\dot{\omega} /|\nabla T|)_{\text {lam }}$, the flame surface area explains the differences in turbulent flame speeds seen earlier in Fig. 4. First, for the unity Lewis number simulations, since the turbulent to laminar source term ratio is close to unity for all cases, the change in flame speeds can be attributed entirely to a change in flame surface area. Second, the difference between the non-unity and unity Lewis number simulations at the same $\mathrm{Ka}_{\delta}$ are due to the difference in $\left\langle\dot{\omega} / \dot{\omega}_{\text {lam }}\right\rangle$ since, for a given $\mathrm{Ka}_{\delta}$, the flame surface area is roughly constant.

In order to understand and explain the differences and similarities in turbulent flame speeds reported in Fig. 4, the geometry of the reaction zone and the mean fuel consumption rate $\dot{\omega}_{F}$ are investigated in detail in the next sections.

\section{Geometry of reaction zone}

In this section, the effects of fuel chemistry, equivalence ratio, and differential diffusion on the distributions of tangential strain rate and curvature at the reaction surface are studied. These quantities are related to both the turbulent flame surface area and chemical source terms as stretching, straining, and curvature can affect the chemical source terms and cause small-scale wrinkling of the flame surface. 
The strain rate tangential to the reaction surface is computed as

$$
a_{t}=\nabla \cdot \mathbf{u}-\mathbf{n} \cdot \nabla \mathbf{u} \cdot \mathbf{n}
$$

where $\mathbf{n}=-\nabla T /|\nabla T|$. The strain rate fields are computed over the entire domain. The conditional probability density functions taken on the isotherm $T=T_{\text {peak }}$ for all cases are presented in Fig. 8a. The stain rate distributions are normalized by the respective Kolmogorov time scales at the reaction zone. Another possible normalization would use the flame time scale $\left(\tau_{F}=l_{F} / S_{L}\right)$. However, at high Karlovitz numbers, it was shown by Bobbitt et al. [35] that the enstrophy scaled with the local Kolmogorov time scale, $\tau_{\eta}$. Hence, a similar scaling is expected for the tangential strain rate. Once normalized, very similar distributions of strain rate are observed for the different cases independently of the reaction zone Karlovitz numbers, chemistry, and transport effects. This indicates that the strain rate is only affected by the local turbulent conditions and the choice of isotherm does not affect the normalized distributions. It should be noted that $\tau_{\eta}$ varies by orders of magnitude between the different cases (from $\tau_{\eta}=4.6 \cdot 10^{-5} \mathrm{~s}^{-1}$ in case $\mathrm{A}_{n \mathrm{C} 7}$ to $\tau_{\eta}=2.5 \cdot 10^{-7} \mathrm{~s}^{-1}$ in case $\mathrm{D}_{n C 7}$. It is also important to note that approximately $80 \%$ of the strain rate is positive, as previously reported by Savard and Blanquart [17]. This is consistent with the analysis of Yeung et al. [51] who reported that, when the propagation speed is much smaller than the Kolmogorov speed $\left(S_{L} / u_{\eta} \ll 1\right)$, propagating and material surfaces present the same strain rate distribution where $80 \%$ of the strain rate is positive.

The curvature is defined as:

$$
\kappa=-\nabla \cdot \mathbf{n}
$$

It is taken to be positive when the center of curvature is in the burnt gases. Probabil- 
ity density functions of curvature at the reaction zone, normalized by their respective standard deviations, are shown in Fig. $8 b$. The means of all distributions are close to zero and the normalized distributions have very similar shapes approaching symmetry around $\kappa=0$. The standard deviations of all cases, normalized by the fuel reaction zone thickness and by the Kolmogorov length scale, are shown in Figs. $9 \mathrm{a}$ and $9 \mathrm{~b}$ respectively. When normalized by the reaction zone thickness, $\sigma_{\kappa}$ increases by a factor of 10 between the lowest and highest Karlovitz numbers and radii smaller than the laminar reaction zone thicknesses are observed in all cases. Such small curvatures are typical of flames in the thin and distributed reaction zone regimes. As noted by Peters [1], a scaling of the curvature with the Gibson scale is expected in the corrugated flamelet regime but not in the thin and distributed reaction zones regimes since the Gibson scale would be smaller than the Kolmogorov length scale. A scaling of $\kappa$ with $\eta$ may thus be expected as it is the smallest length scale. This is shown in Fig. 9b and the change in $\sigma_{\kappa}$ with $\mathrm{Ka}_{\delta}$ is much less (a factor of two at most). This indicates that the curvature is mostly influenced by the Kolmogorov length scale. There may still be residual effects of the reaction zone thickness due to the fact the the reaction zone is broadened in cases C and D. Regardless of the dependence of $\kappa$ on the Karlovitz number, at a given reaction zone Karlovitz number, no chemistry, equivalence ratio, or differential diffusion effects are observed on the distributions of curvature.

[Fig. 8 about here.]

[Fig. 9 about here.]

These results clearly underscore that, in the present detailed chemistry simulations, fuel, chemical mechanisms, equivalence ratios, and differential diffusion do not affect the reaction zone geometry as the distributions of strain rate and curvature 
are essentially unchanged. This is consistent with the results of Savard and Blanquart [17] and the analysis of Yeung et al. [51]. At high Karlovitz numbers, the reaction zone surfaces behave as material surfaces. This explains why, at a given reaction zone Karlovitz number and integral length scale, similar flame surface areas are observed despite the differences in chemistry and transport (Fig. 7a).

\section{Chemical source terms}

In this section, the effects of fuel chemistry, equivalence ratio, and differential diffusion on the fuel consumption rates are investigated. Only the fuel consumption rates are studied, but it should be noted that heat release rates present very similar trends, as was shown by Lapointe et al. [18] for the present $n$-heptane turbulent flames.

Recall that two main observations were made from the conditional means of the fuel consumption rate at the reaction surface (Fig. 7b). First, the mean source term of any of the unity Lewis number turbulent flames is very close to that of the corresponding laminar flame. This indicates that, for all fuels, turbulence does not affect significantly the mean fuel consumption rate in the absence of differential diffusion. Second, simulations including differential diffusion consistently exhibit a lower mean source term than the corresponding laminar flame with differential diffusion. Yet, the conditional mean approaches that of the laminar flame without differential diffusion as $\mathrm{Ka}_{\delta}$ is increased.

In this section, the unity and non-unity Lewis number cases are investigated separately to isolate the effects of differential diffusion. 


\subsection{Unity Le: effects of dissipation rate}

Several correlations have been proposed in the literature for the fluctuations of the chemical source terms, including curvature and strain rate [23, 52]. However, it was shown by Savard and Blanquart [17] that, in high Karlovitz number unity Lewis flames, the burning rate does not correlate strongly with either strain rate or curvature.

Kolla [53] showed that if the species mass fractions are only a function of the progress variable, the species transport equation can take the following form:

$$
\dot{\omega}_{c} \frac{\partial Y_{i}}{\partial c}=\frac{\rho \chi}{2} \frac{\partial^{2} Y_{i}}{\partial c^{2}}+\dot{\omega}_{i}
$$

where $Y_{i}$ is the species mass fraction, $c$ is the progress variable, and $\chi$ is its dissipation rate defined as:

$$
\chi=2 \alpha|\nabla c|^{2} .
$$

Recently, Savard [27] derived the same premixed flamelet equations by applying a local coordinate transformation to the species and temperature transport equations. These results underscore that the fuel burning rate for unity Lewis number flames in the thin reaction zone regime is a function of a progress variable (e.g. the temperature) and its dissipation rate and suggest a linear dependence of the chemical source term on $\chi$. In other words, at a fixed temperature/progress variable, the fluctuations in fuel consumption are strongly correlated with $\chi$.

This is illustrated in Fig. 10 presenting the joint probability density functions of the fuel consumption rate $v s$. the dissipation rate evaluated at $T_{\text {peak }}$ for different fuels in case B. A strong correlation is observed for the three fuels used in this study, showing a close-to-linear dependence. The correlation coefficients for the different 
cases are defined as

$$
r=\frac{\sum_{i=1}^{n}\left(X_{i}-\bar{X}\right)\left(Y_{i}-\bar{Y}\right)}{\sqrt{\sum_{i=1}^{n}\left(X_{i}-\bar{X}\right)^{2}} \sqrt{\sum_{i=1}^{n}\left(Y_{i}-\bar{Y}\right)^{2}}}
$$

and are summarized in Table 5. The coefficients are close to unity, especially for cases $\mathrm{A}$ and $\mathrm{B}$, confirming the strong linear relationship between $\dot{\omega}_{F}$ and $\chi$. Figure 11 shows the joint probability density functions of the fuel consumption rate $v s$. the dissipation rate evaluated at $T_{\text {peak }}$ for different fuels in case $\mathrm{C}$. Once again, a good correlation and linear dependence are observed for the different fuels but weaker than for case B. This is most likely due to the widening of the reaction zone at increasing Karlovitz numbers. Lapointe et al. [18] showed that the reaction zone thicknesses for the $n$-heptane flames were close to the laminar values in cases A and $\mathrm{B}$ and larger in cases $\mathrm{C}$ and $\mathrm{D}$. The validity of the flamelet equations, Eq. 24, relies on the assumption that the reaction zone is thin. Therefore, a weaker correlation between $\dot{\omega}_{F}$ and $\chi$ is expected in the distributed reaction zone regime.

[Fig. 10 about here.]

[Fig. 11 about here.]

[Table 5 about here.]

Given the strong dependence of the source term on the scalar dissipation rate, it is important to analyze its evolution. To provide more information, the PDFs of the logarithm of $\chi$ on the reaction surface are shown in Fig. 12a. The distribution of dissipation rate of a passive scalar with a uniform mean gradient in homogeneous isotropic turbulence (HIT), taken from Vedula et al. [54], is also shown for comparison purposes. When normalized by their respective means and standard deviations, very similar distributions are observed for all fuels and Karlovitz numbers. In fact, 
the distributions are the same as that from HIT and are close to a log-normal distribution, which is characteristic of turbulent mixing of passive scalars [55-57]. The standard deviations of the logarithm of $\chi$ are shown in Fig. 12b. As for the mixing of a passive scalar, the standard deviations increase with increasing Reynolds number $\left(\operatorname{Re}_{\lambda}\right.$ is computed using the viscosity at $\left.T_{\text {peak }}\right)$. More specifically, for the present objectives, they do not show strong chemistry effects. Finally, the means of the dissipation rate are shown in Fig. 13. For cases A and B, the mean dissipation rate $\langle\chi\rangle$ is close to $\chi_{\text {lam. }}$. An increase above $\chi_{\text {lam }}$ is observed for cases $\mathrm{C}$ and D. In other words, the mean of the distribution is related to fuel specific, laminar quantities while the standard deviation is entirely due to turbulence.

[Fig. 12 about here.]

[Fig. 13 about here.]

Now, the two previous results are used to explain burning rates shown in Fig. $7 \mathrm{~b}$. Assuming that, at a fixed temperature, the fuel consumption rate is solely a function of the dissipation rate, the conditional mean fuel consumption rate can be expressed as

$$
\langle\dot{\omega} \mid T\rangle=\int \dot{\omega}(T, \chi) \mathrm{P}(\chi \mid T) \mathrm{d} \chi
$$

where $\mathrm{P}(\chi \mid T)$ is the probability density function of the dissipation rate at the given temperature and $\dot{\omega}(T, \chi)$ is the functional dependence of the burning rate on $\chi$. Based on the results shown in Figs. 10 and 11, this function is approximated by expanding around the laminar dissipation rate

$$
\dot{\omega}(T, \chi)=\dot{\omega}\left(T, \chi_{\mathrm{lam}}\right)+\beta\left(\chi-\chi_{\mathrm{lam}}\right)+\mathcal{O}\left(\chi^{2}\right) .
$$

Substituting Eq. 28 into Eq. 27, keeping only the linear term (since the dependence 
of $\dot{\omega}$ on $\chi$ is almost linear $)$, and observing that $\dot{\omega}\left(T, \chi_{\text {lam }}\right) \approx \dot{\omega}_{\text {lam }}$ yields

$$
\langle\dot{\omega} \mid T\rangle \approx \dot{\omega}_{\mathrm{lam}}+\beta\left(\langle\chi\rangle-\chi_{\mathrm{lam}}\right)
$$

Thus, if the first moment of the probability density function is close to the laminar dissipation rate

$$
\langle\chi\rangle=\int \chi \mathrm{P}(\chi) \mathrm{d} \chi \approx \chi_{\mathrm{lam}}
$$

as seen in Fig. 13, then the mean fuel consumption rate should be close to the laminar value and $\langle\dot{\omega} \mid T\rangle \approx \dot{\omega}_{\text {lam. }}$ This was observed in Fig. 7b.

It should be noted that, in Eq. 28, all the effects of chemistry are contained in $\dot{\omega}\left(T, \chi_{\mathrm{lam}}\right)$ and $\beta$. More specifically, these parameters take different values for different fuels, equivalence ratios, unburnt temperature, etc. Nevertheless, as shown previously, this does not affect the conclusions made here, namely that $\langle\dot{\omega} \mid T\rangle \approx$ $\dot{\omega}_{\text {lam. }}$. In cases $\mathrm{C}$ and $\mathrm{D},\langle\chi\rangle$ being larger than $\chi_{\text {lam }}$ is consistent with the increase in the conditional means of normalized fuel consumption rates at $T_{\text {peak }}$ shown in Fig. $7 b$.

\subsection{Non-unity Le}

As opposed to the unity Lewis number cases, the chemical source terms are not solely a function of a progress variable and its dissipation rate when differential diffusion is considered. The correlation between the fuel consumption rate and the dissipation rate is still present but much weaker [27] and does not explain the trends observed in Fig. $7 b$.

The first important observation is that the mean fuel consumption rate is lower than in the corresponding unity Lewis number flame. This is observed for all fuels, 
equivalence ratios, and mechanisms, but to a lesser extent in the methane flame. This reduction in fuel consumption was previously related to curvature effects. More precisely, Savard and Blanquart [17] showed that, in case $\mathrm{B}_{n \mathrm{C} 7}$, low fuel consumption rates are correlated with regions of high curvature, suggesting the presence of focusing/defocusing effects. This is due to the above unity Lewis number of the present fuels. An increased burning rate has been observed for fuels with lesser than unity Lewis numbers such as hydrogen [12].

Additionally, Savard and Blanquart [17] showed that the fuel ( $n$-heptane) mass fraction was strongly correlated with curvature, more so than radicals $\mathrm{H}$ and $\mathrm{OH}$. Therefore, effects of curvature on the burning rate are felt through the fuel mass fraction. This explains why, in the present study, different fuels with similar (above unity) Lewis number exhibit similar reductions in mean fuel consumption rates. This result is specific to fuels with significantly non-unity Lewis numbers and contrasts with common observations made in methane flames. For instance, in twodimensional simulations of methane/air premixed flames, Echekki and Chen [58] reported a stronger correlation between the hydrogen atom mass fraction and curvature. These results can explain the present reduction in the mean fuel consumption rate compared to the laminar flame (for fuels with greater than unity Lewis numbers). Since the Lewis number of the fuel (methane) is very close to unity, focusing/defocusing differential diffusion effects are still present through radicals such as $\mathrm{H}$ but to a lesser extent than in high Lewis number fuels.

It was shown in Fig. 9a that curvatures get stronger with increasing $\mathrm{Ka}_{\delta}$. Hence, a decrease in the mean fuel consumption rate due to larger curvatures (and thus stronger focusing/defocusing effects) could be expected. This is what happens for moderate Karlovitz numbers up to cases A and B. However, as the Karlovitz number is further increased towards cases $\mathrm{C}$ and $\mathrm{D}$, differential diffusion effects are di- 
minished with increasing turbulent mixing (effective Lewis numbers tend towards unity as the Karlovitz number increases $[18,59])$. The fuel consumption rates are then less sensitive to curvature and the effective mean values increase towards the unity Lewis number values.

\subsection{Chemical mechanism}

Figure 14 presents the mean fuel consumption rates for cases $\mathrm{B}$ and $\mathrm{C}$ with $n$ heptane using different mechanisms. In the laminar flames of case B (Fig. 14a), the reduced mechanism predicts consumption rates very similar to the detailed CaltechMech, for both non-unity and unity Lewis number flames. This is expected as the reduced model was derived from the full CaltechMech [39]. In the turbulent flames (Fig. 14b), both mechanisms again predict very similar fuel consumption rates. It is important to note that the effect of differential diffusion is significantly larger than the difference between the two chemical models. The flame-turbulence interaction is thus not affected by the size of the chemical mechanism.

The laminar flames of case $\mathrm{C}$ (Fig. 14c) do show some slight difference in the fuel consumption rates between the reduced mechanism and the detailed JetSurF mechanism. This could be due to some inherent differences between the models as JetSurF has isomers for all radicals and a large number of reactions dedicated to thermal cracking and $\beta$-scissions. Despite these differences, in the turbulent flames (Fig. 14d), the fuel consumption rates are very close once normalized by their respective laminar peak values. This indicates that, as for case $\mathrm{B}$, there is no effect of the mechanism on the turbulent flame behavior. In summary, in both cases B and $\mathrm{C}$, the reduction in fuel consumption rate in the non-unity Lewis number flames is predicted by all three mechanisms and the rates of the unity Lewis number flames 
remain within approximately $10 \%$ of the laminar values.

[Fig. 14 about here.]

\section{Summary: controlling parameters}

The present results suggest that the mean turbulent flame speed and fuel consumption rates can be predicted with the knowledge of only a few global laminar flame properties. If these properties are predicted accurately with any given chemical model, the results from simulations of turbulent flames are likely to also be accurate.

More precisely, when appropriately normalized, all relevant quantities follow universal behaviors independent of the fuels, equivalence ratios, chemical mechanisms, etc. Some of the quantities are entirely controlled by turbulence and do not require knowledge of the underlying chemistry:

- strain rate scales with $\tau_{\eta}$ (Fig. 8a),

- standard deviation of $\ln (\chi)$ depends on $\operatorname{Re}_{\lambda}$ (Fig. 12b).

Some quantities are entirely controlled by chemical quantities:

- mean of $\chi$ follows closely $\chi_{\text {lam }}$ (Fig. 13),

- mean $\dot{\omega}_{F}$ scales with $\dot{\omega}_{F, \text { lam }}\left(T_{\text {peak }}\right)$ for unity Lewis numbers (Fig. 7b).

Finally, some quantities are controlled by both chemical and turbulent quantities:

- curvature normalized by $\eta_{\delta}$ is a function of $\mathrm{Ka}_{\delta}$ (Fig. 9b),

- turbulent surface area is a function of $\mathrm{Ka}_{\delta}$ (Fig. 7a) (and most likely $l / l_{F}$ ),

- mean $\dot{\omega}_{F}$ normalized by $\dot{\omega}_{F, \text { lam }}\left(T_{\text {peak }}\right)$ is only function of $\operatorname{Le}_{F}$ and $\mathrm{Ka}_{\delta}$ (Fig. 7b), 
- turbulent flame speed normalized by $S_{L}$ is a function of $\mathrm{Ka}_{\delta}$ (Fig. 4) (and most likely $\left.l / l_{F}\right)$.

In summary, the relevant chemical quantities are the same as used in the Peters/Borghi regime diagram, namely

- laminar flame speed $\left(S_{L}\right)$,

- laminar flame thickness $\left(l_{F}\right)$,

- reaction zone thickness $\left(\delta_{F}\right)$,

with the addition of the fuel Lewis number to capture differential diffusion effects. The peak chemical source term is also used in the various normalizations, but its value can be expressed in terms of the above three quantities.

\section{Conclusion}

A series of direct numerical simulations of premixed flames at different Karlovitz numbers have been performed using detailed chemistry. Fuels, equivalence ratios, chemical models, and turbulent intensities were systematically varied. The analysis focused on turbulent flame speeds, chemical source terms, reaction zone surface areas, and geometry of the reaction zones. Differential diffusion effects have been systematically isolated by performing simulations with both non-unity and unity Lewis numbers. It should be noted that only fuels with above unity Lewis numbers were considered. Extension to fuels with below unity Lewis numbers $\left(\operatorname{Le}_{F} ; 1\right)$ or thermo-diffusively unstable conditions should be the subject of future work.

First and foremost, in the absence of differential diffusion, all fuels still presented similar normalized turbulent flame speeds at a fixed reaction zone Karlovitz num- 
ber. When differential diffusion effects were included, all fuels presented similar turbulent flame speeds, yet lower than their unity Lewis number counterparts. This is due to the above unity Lewis number of the present fuels. Enhanced burning velocities are expected for light fuels such as hydrogen. Second, distributions of tangential strain rate and curvature on the reaction zone surface were essentially the same when normalized by the Kolmogorov scales at the reaction zone, illustrating that chemistry and transport effects do not alter the geometry of the reaction zone. Third, differential diffusion effects on the chemical source terms were present even at high Karlovitz numbers. In the unity Lewis number simulations, all fuels presented a mean fuel consumption rate close to the respective laminar value. In the non-unity Lewis number simulations, lower mean burning rates were observed. All three heavy hydrocarbon fuels ( $n$-heptane, iso-octane, and toluene) presented those trends but the mean fuel consumption rate of the methane flame was higher, highlighting the importance of the fuel Lewis number.

In summary, fuel effects in the present high Karlovitz premixed flames were minor. Turbulent flame behavior can be predicted with the knowledge of only a few global laminar flame properties $\left(S_{L}, l_{F}, \delta_{F}, \mathrm{Le}_{i}\right)$ and turbulence characteristics. Differential diffusion and changes in turbulence intensity at the reaction zone affect the turbulent flame speed significantly more than different fuels, equivalence ratios, and chemical mechanisms. 


\section{A Turbulent flame speed correlation}

The volumetric integral of the fuel consumption rate in Eq. 13 is rewritten as a surface integral along the isocontour $T=T_{\text {peak }}$ and an integral in the normal direction:

$$
\int_{V} \dot{\omega}_{F} \mathrm{~d} V=\int_{T=T_{\text {peak }}}\left(\int \dot{\omega}_{F} \mathrm{~d} n\right) \mathrm{d} A
$$

where the local consumption rate $\int \dot{\omega}_{F} \mathrm{~d} n$ can be rewritten using $\mathrm{d} n=\frac{\mathrm{d} T}{|\nabla T|}$

$$
\int \dot{\omega}_{F} \mathrm{~d} n=\int \dot{\omega}_{F} \frac{\mathrm{d} T}{|\nabla T|}
$$

Then, the observation of Savard and Blanquart [17] that the fuel consumption locally scales like its value at $T=T_{\text {peak }}$ is used:

$$
\dot{\omega}_{F}(T) \approx \dot{\omega}_{F, \text { lam }}(T) \frac{\dot{\omega}_{F}\left(T_{\text {peak }}\right)}{\dot{\omega}_{F, \text { lam }}\left(T_{\text {peak }}\right)}
$$

and a similar assumption is made for the gradient of temperature:

$$
|\nabla T|(T) \approx|\nabla T|_{\text {lam }}(T) \frac{|\nabla T|\left(T_{\text {peak }}\right)}{|\nabla T|_{\text {lam }}\left(T_{\text {peak }}\right)}
$$

Combining Eqs. 13, A.1, A.2, A.3, and A.4, the turbulent flame speed can be approximated as:

$$
\frac{S_{T}}{S_{L}} \approx \frac{A_{T}}{A} \frac{\left\langle\dot{\omega}_{F} /|\nabla T| \mid T_{\text {peak }}\right\rangle}{\dot{\omega}_{F, \text { lam }} /\left|\nabla T_{\text {lam }}\right|} .
$$

$\left\langle\dot{\omega}_{F} /|\nabla T| \mid T_{\text {peak }}\right\rangle$ is the conditional mean, at $T=T_{\text {peak }}$, of the fuel consumption rate divided by the temperature gradient (both $\dot{\omega}_{F}$ and $\nabla T$ are computed locally at each grid cell). $A=L^{2}$ is the domain cross-section, and $\dot{\omega}_{F, \text { lam }} /\left|\nabla T_{\text {lam }}\right|$ is computed at $T=T_{\text {peak }}$ in the corresponding laminar flame. 
It is important to note that, as opposed to Savard and Blanquart [17], it was not assumed that the temperature gradient at the reaction zone is equal to its laminar counterpart. The improved correlation from assuming Eq. A.4 rather $|\nabla T|)(T) \approx$ $|\nabla T|_{\operatorname{lam}}(T)$ is seen by comparing Fig. 15 to Fig. 6 . The correlation between $S_{T} / S_{L}$ and $\frac{A_{T}}{A} I_{0}$ is stronger in Fig. 6. This is quantified by computing the $\mathrm{L}^{2}$-norm of the prediction error made on the flame speed

$$
\epsilon_{S_{T}}=\sqrt{\frac{\sum\left[\left(S_{T} / S_{L}\right)-\left(A_{T} I_{0} / A\right)\right]^{2}}{\sum\left(S_{T} / S_{L}\right)^{2}}}
$$

for both Figs. 6 and 15. The error is reduced from $13 \%$ to $7 \%$ by including the changes in temperature gradient.

[Fig. 15 about here.]

\section{Acknowledgements}

The authors gratefully acknowledge funding from Air Force Office of Scientific Research (FA9550-12-1-0472 and FA9550-12-1-0144) under supervision of Dr. Chiping Li and Fonds de Recherche du Québec - Nature et Techonologies for financial support. This research used resources of the National Energy Research Scientific Computing Center, a DOE Office of Science User Facility supported by the Office of Science of the U.S. Department of Energy under Contract No. DE-AC0205CH11231. This work used the Extreme Science and Engineering Discovery Environment (XSEDE), which is supported by National Science Foundation grant number ACI-1053575. 


\section{References}

[1] N. Peters, The turbulent burning velocity for large-scale and small-scale turbulence, J. Fluid Mech. 384 (1999) 107 - 132.

[2] N. Peters, Turbulent Combustion, Cambridge University Press, 2000.

[3] H. Pitsch, Large-eddy simulation of turbulent combustion, Ann. Rev. Fluid Mech. 38 (2006) $453-482$.

[4] Y.-C. Chen, R. Bilger, Experimental investigation of three-dimensional flame-front structure in premixed turbulent combustion: Ii. lean hydrogen/air bunsen flames, Combust. Flame 138 (2004) 155 - 174.

[5] J. Hult, S. Gashi, N. Chakraborty, M. Klein, K. Jenkins, S. Cant, C. Kaminski, Measurement of flame surface density for turbulent premixed flames using plif and dns, Proc. Combust. Inst. 31 (2007) 1319 - 1326.

[6] M. Dunn, A. Masri, R. Bilger, A new piloted premixed jet burner to study strong finite-rate chemistry effects, Combust. Flame 151 (2007) 46- 60.

[7] M. Dunn, A. Masri, R. Bilger, R. Barlow, G. Wang, The compositional structure of highly turbulent piloted premixed flames issuing into a hot coflow, Proc. Combust. Inst. 32 (2009) $1779-1786$.

[8] F. Carbone, J. Smolke, A. Fincham, F. Egolfopoulos, Characteristics of piloted premixed turbulent-jet flames of methane and $\mathrm{c}_{6}-\mathrm{c}_{8}$ hydrocarbons, WSSCI Spring 2014 Meeting (2014) paper 14S-03.

[9] A. Aspden, J. Bell, M. Day, S. Woosley, M. Zingale, Turbulence-flame interactions in type ia supernovae, Astrophys. J. 689 (2008) 1173 - 1185.

[10] A. Aspden, J. Bell, S. Woosley, Distributed flames in type ia supernovae, Astrophys. J. 710 (2010) $1654-1663$.

[11] A. Aspden, M. Day, J. Bell, Turbulence-flame interactions in lean premixed hydrogen: transition to the distributed burning regime, J. Fluid Mech. 680 (2011) $287-320$.

[12] A. Aspden, M. Day, J. Bell, Turbulence-chemistry interaction in lean premixed hydrogen combustion, Proc. Combust. Inst. 35 (2015) 1321-1329.

[13] H. Wang, K. Luo, J. Fan, Direct numerical simulation and conditional statistics of hydrogen/air turbulent premixed flames, Energ. Fuel 27 (2013) 549 - 560.

[14] A. Aspden, M. Day, J. Bell, Lewis number effects in distributed flames, Proc. Combust. Inst. 33 (2011) $1473-1480$.

[15] R. Sankaran, E. Hawkes, J. Chen, T. Lu, C. Law, Structure of a spatially developing turbulent lean methane-air bunsen flame, Proc. Combust. Inst. 31 (2007) 1291 - 1298.

[16] B. Savard, B. Bobbitt, G. Blanquart, Structure of a high karlovitz n-c7h16 premixed turbulent flame, Proc. Combust. Inst. 35 (2015) 1377-1384.

[17] B. Savard, G. Blanquart, Broken reaction zone and differential diffusion effects in a high karlovitz n-c7h16 premixed turbulent flame, Combust. Flame 162 (2015) 2020 2033.

[18] S. Lapointe, B. Savard, G. Blanquart, Differential diffusion effects, distributed burning, and local extinctions in high karlovitz premixed flames, Combust. Flame 162 (2015) $3341-3355$.

[19] A. Poludnenko, E. Oran, The interaction of high-speed turbulence with flames: Global properties and internal flame structure, Combust. Flame 157 (2010) 995 - 1011.

[20] A. Poludnenko, E. Oran, The interaction of high-speed turbulence with flames: Turbulent flame speed, Combust. Flame 158 (2011) $301-326$.

[21] A. Poludnenko, Pulsating instability and self-acceleration of fast turbulent flames, Phys. Fluids 27 (014106) (2015) $1-25$.

[22] H. Carlsson, R. Yu, X.-S. Bai, Flame structure analysis for categorization of lean premixed ch4/air and h2/air flames at high karlovitz numbers: Direct numerical simulation studies, Proc. Combust. Inst. 35 (2015) 1425-1432.

[23] R. Sankaran, E. Hawkes, C. Yoo, J. Chen, Response of flame thickness and propagation speed under intense turbulence in spatially developing lean premixed methane-air jet flames, Combust. Flame 162 (2015) $3294-3306$.

[24] T. Dunstan, N. Swaminathan, K. Bray, R. Cant, Geometrical properties and turbulent flame speed measurements in stationary premixed v-flames using direct numerical simulations, Flow Turbul. Combust. 87 (2011) 237-259.

[25] H. Kolla, E. Hawkes, A. Kerstein, N. Swaminathan, J. Chen, On velocity and reactive scalar spectra in turbulent premixed flames, J. Fluid Mech. 754 (2014) 456-487. 
[26] S. Pope, Turbulent Flows, Cambridge University Press, 2000.

[27] B. Savard, Characterization and modeling of premixed turbulent n-heptane flames in the thin reaction zone regime, Ph.D. thesis, California Institute of Technology (2015).

[28] B. Savard, Y. Xuan, B. Bobbitt, G. Blanquart, A computationally-efficient, semiimplicit, iterative method for the time-integration of reactive flows with stiff chemistry, J. Comp. Phys. 295 (2015) 740-769.

[29] O. Desjardins, G. Blanquart, G. Balarac, H. Pitsch, High order conservative finite difference scheme for variable density low mach number turbulent flows, J. Comp. Phys. 227 (2008) $7125-7159$.

[30] C. Pierce, Progress-variable approach for large-eddy simulation of turbulent combustion, Ph.D. thesis, Stanford University (2001).

[31] M. Herrmann, G. Blanquart, V. Raman, Flux corrected finite volume scheme for preserving scalar boundedness in reacting large-eddy simulations, AIAA Journal 44 (2006) $2879-2886$.

[32] C. Rosales, C. Meneveau, Linear forcing in numerical simulations of isotropic turbulence: Physical space implementations and convergence properties, Phys. Fluids 17 (2005) $0951061-8$.

[33] P. Carroll, G. Blanquart, The effect of velocity field forcing techniques on the nature and evolution of the karman-howarth equation, J. Turbul. 15 (2014) $429-448$.

[34] T. Lundgren, Linearly forced isotropic turbulence, in: Annual Research Briefs (Center for Turbulence Research), Stanford, 2003, 2003, pp. 461-473.

[35] B. Bobbitt, S. Lapointe, G. Blanquart, Vorticity transformation in high karlovitz number premixed flames, Phys. Fluids 28 (2016) 015101.

[36] G. Smith, D. Golden, M. Frenklach, N. W. Moriarty, B. Eiteneer, M. Goldenberg, C. T. Bowman, R. K. Hanson, S. Song, W. C. Gardiner, V. V. Lissianski, Z. Qin, Gri-mech 3.0. Available at http://www.me.berkeley.edu/gri_mech/. (Accessed: Jan. 2015).

[37] G. Blanquart, Caltechmech 2.1. Available at http://theforce.caltech.edu/resources/. (Accessed: Jan. 2015).

[38] H. Wang, E. Dames, B. Sirjean, D. A. Sheen, R. Tango, A. Violi, J. Y. W. Lai, F. N. Egolfopoulos, D. F. Davidson, R. K. Hanson, C. T. Bowman, C. K. Law, W. Tsang, N. P. Cernansky, D. L. Miller, R. P. Lindsted, A high-temperature chemical kinetic model of n-alkane (up to n-dodecane), cyclohexane, and methyl-, ethyl-, n-propyl and n-butyl-cyclohexane oxidation at high temperatures, jetsurf version 2.0, (http://web.stanford.edu/group/haiwanglab/jetsurf/jetsurf2.0/index.html) (2010).

[39] F. Bisetti, G. Blanquart, M. Mueller, H. Pitsch, On the formation and early evolution of soot in turbulent nonpremixed flames, Combust. Flame 159 (2012) 317 - 335.

[40] O. Hirschfelder, C. Curtiss, R. Bird, Molecular Theory of Gases and Liquids, John Wiley and Sons, New York, 1954.

[41] C. Wilke, A viscosity equation for gas mixtures, Journal of Chemical Physics 18 (4) (1950) 517-519.

[42] A. Eucken, The heat-carrying capabilities, the specific heat, and the internal friction of gas, Physik Z. 14 (1913) 324-333.

[43] S. Mathur, P. Tondon, S. Saxena, Thermal conductivity of binary, ternary and quaternary mixture of rare gases, Molecular Physics 12 (6) (1967) 569-579.

[44] H. Pitsch, FlameMaster: A C++ computer program for 0D combustion and 1D laminar flame calculations. Available at http://www.itv.rwthaachen.de/downloads/flamemaster/. (1998).

[45] N. Burali, S. Lapointe, B. Bobbitt, G. Blanquart, Y. Xuan, Assessment of the constant non-unity lewis number assumption in chemically reacting flows, Combust. Theor. Model. (2016) in revision.

[46] S. Menon, P. Boettcher, G. Blanquart, Enthalpy based approach to capture heat transfer effects in premixed combustion, Combust. Flame 160 (2013) 1242-1253.

[47] G. Damkohler, Der einfluss der turbulenz auf die flammengeschwindigenkeit in gasgemishcen, Z. Elektrochem 46 (1940) $601-652$.

[48] K. Bray, Studies of the turbulent burning velocity, Proc. Roy. Soc. London 431 (1990) 313-323.

[49] S. Candel, T. Poinsot, Flame stretch and the balance equation for the flame area, Combust. Sci. Technol. 70 (1990) 1-15.

[50] W. Lorensen, H. Cline, Marching cubes: A high resolution 3d surface construction algorithm, Computer Graphics 21. 
[51] P. Yeung, S. Girimaji, S. Pope, Straining and scalar dissipation on material surfaces in turbulence: implications for flamelets, Combust. Flame 79 (1990) 340 - 365.

[52] A. Amato, M. Day, R. Cheng, J. Bell, T. Lieuwen, Leading edge statistics of turbulent, lean, $\mathrm{h}_{2}$-air flames, Proc. Combust. Inst. 35 (2015) 1313-1320.

[53] H. Kolla, Scalar disspation rate based flamelet modelling of turbulent premixed flames, Ph.D. thesis, University of Cambridge (2009).

[54] P. Vedula, P. Yeung, R. Fox, Dynamics of scalar dissipation in isotropic turbulence: a numerical and modelling study, J. Fluid Mech. 433 (2001) 29-60.

[55] E. Effelsberg, N. Peters, Scalar dissipation rates in turbulent jets and jet diffusion flames, Symp. (Int.) Combust. 22 (1989) 693-700.

[56] R. Antonia, K. Sreenivasan, Lognormality of temperature dissipation in a turbulent boundary layer, Phys. Fluids 20 (11) (1977) 1800-1804.

[57] C. Pantano, S. Sarkar, F. Williams, Mixing of a conserved scalar in a turbulent reacting shear layer, J. Fluid Mech. 481 (2003) 291-328.

[58] T. Echekki, J. Chen, Unsteady strain rate and curvature effects in turbulent premixed methane-air flames, Combust. Flame 106 (1996) $184-202$.

[59] B. Savard, G. Blanquart, An a priori model for the effective species lewis numbers in premixed turbulent flames, Combust. Flame 161 (2014) 1547 - 1557. 


\section{List of Figures}

1 Schematic diagram of the flow configuration, from Savard et al. [16].

2 Regime diagram showing current simulations.

3 Turbulent flame speed for case $\mathrm{B}_{n \mathrm{C} 7, \phi 1.3,1}$. Solid black line is the instantaneous flame speed (Eq. 13), red dots indicate snapshots used for the statistics (Eq. 16).

$4 \quad$ Mean turbulent flame speeds. Filled and empty symbols correspond to non-unity and unity Lewis number simulations, respectively. Dashed lines represent power law fits (see text).

5 Normalized turbulent flame speed $\left(S_{T} / S_{L}\right)$, surface area $\left(A_{T} / A\right)$, Eq. 19, and burning efficiency factor $\left(I_{0}\right)$ plotted against time for cases $\mathrm{C}_{\mathrm{ACH} 3}$. Quantities are shown for every snapshot used for the statistics ( $\sim \tau$ apart).

6 Scatter plot of the instantaneous $A_{T}\left\langle\dot{\omega}_{F} /|\nabla T| \mid T_{\text {peak }}\right\rangle /\left(A \dot{\omega}_{F, \text { lam }} /\left|\nabla T_{\text {lam }}\right|\right)$ plotted against the instantaneous normalized turbulent flame speed for each snapshot used for all simulations. Filled and empty symbols correspond to non-unity and unity Lewis number simulations, respectively. The colors refer to different fuels and chemistry models (see Fig. 4).

$7 \quad$ Mean turbulent flame surface areas and normalized fuel consumption rates at the corresponding temperature of peak source term. Filled and empty symbols correspond to non-unity and unity Lewis number simulations, respectively. Filled and empty symbols correspond to non-unity and unity Lewis number simulations, respectively. The colors refer to different fuels and chemistry models (see Fig. 4).

8 Probability density functions of the strain rate and curvature taken on the isosurface of the corresponding temperature of peak source term. The strain rates are normalized by the corresponding Kolmogorov time scales and the curvatures are normalized by their standard deviations. 
9 Standard deviations of curvature on the reaction zone surface, normalized by the fuel reaction zone thickness and by the Kolmogorov length scale, plotted against the reaction zone Karlovitz number. The dashed line on Fig. 9a represents $\sigma_{\kappa} \delta_{F}=0.3 \sqrt{\mathrm{Ka}_{\delta}}$. Filled and empty symbols correspond to non-unity and unity Lewis number simulations, respectively. The colors refer to different fuels and chemistry models (see Fig. 4).

10 Joint probability density functions of the fuel consumption rate and dissipation rate on the isosurface $T=T_{\text {peak }}$ for unity Lewis number cases B.

11 Joint probability density functions of the fuel consumption rate and dissipation rate on the isosurface $T=T_{\text {peak }}$ for unity Lewis number cases $\mathrm{C}$.

12 a) Probability density functions of the dissipation rate taken on the isosurface of the corresponding temperature of peak source term. b) Standard deviations of the PDFs. The dashed line in a) and HIT data in b) correspond to the PDF of dissipation rate of a passive scalar in homogeneous isotropic turbulence from Vedula et al. [54]. Empty symbols correspond to unity Lewis number simulations and the colors refer to different fuels and chemistry models (see Fig. 4).

13 Means of the probability density functions of $\chi$ at $T=T_{\text {peak }}$ plotted against the corresponding laminar dissipation rate. Empty symbols correspond to unity Lewis number simulations and the colors refer to different fuels and chemistry models (see Fig. 4).

14 Conditional means of normalized $n$-heptane consumption rate for three different mechanisms. Solid lines correspond to non-unity Lewis number cases while dashed lines correspond to unity Lewis number cases. The fuel consumption rates in the turbulent flames are normalized by the peak value in the corresponding laminar flame.

15 Scatter plot of the instantaneous $A_{T}\left\langle\dot{\omega}_{F} \mid T_{\text {peak }}\right\rangle /\left(A \dot{\omega}_{F, \text { lam }}\right)$ plotted against the instantaneous normalized turbulent flame speed for each snapshot used for all simulations. Filled and empty symbols correspond to non-unity and unity Lewis number simulations, respectively. The colors refer to different fuels and chemistry models (see Fig. 4). 


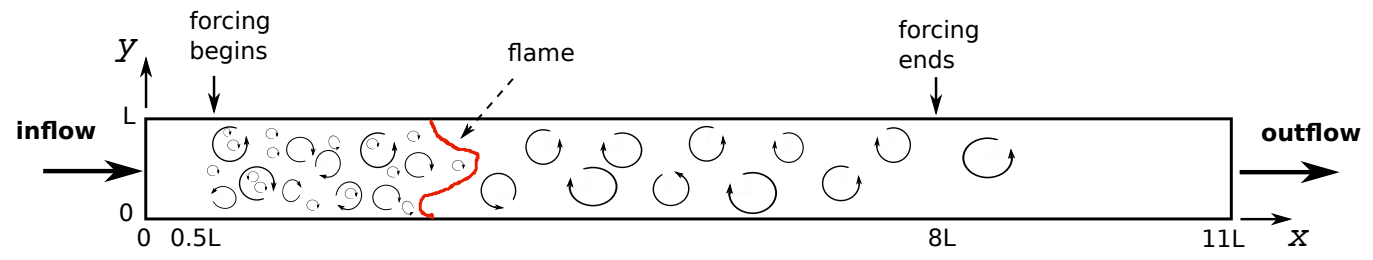

Fig. 1. Schematic diagram of the flow configuration, from Savard et al. [16]. 


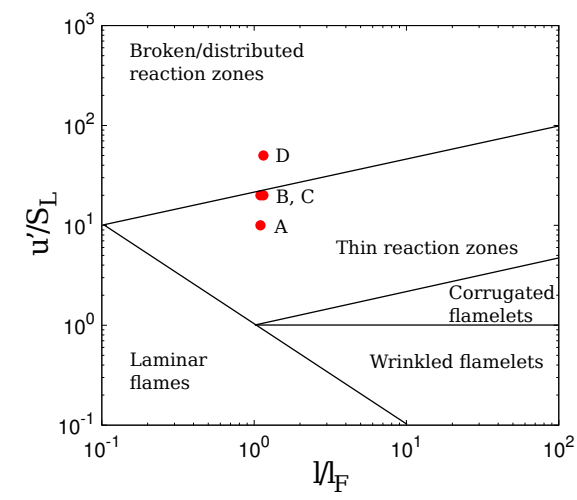

Fig. 2. Regime diagram showing current simulations. 


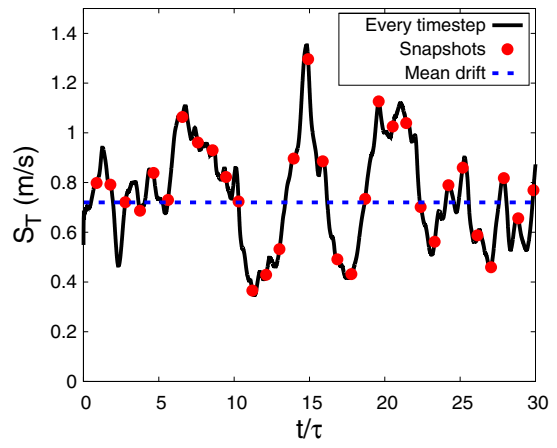

Fig. 3. Turbulent flame speed for case $\mathrm{B}_{n \mathrm{C} 7, \phi 1.3,1}$. Solid black line is the instantaneous flame speed (Eq. 13), red dots indicate snapshots used for the statistics (Eq. 16). 


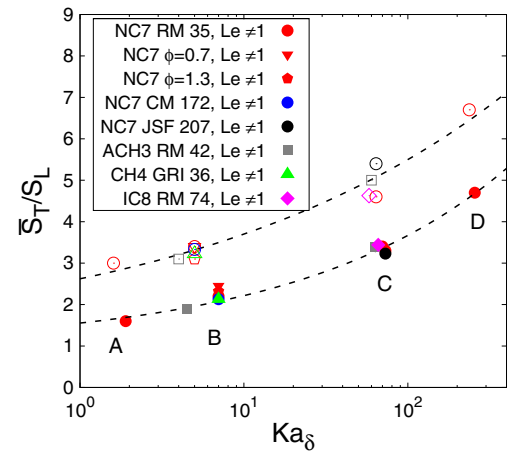

Fig. 4. Mean turbulent flame speeds. Filled and empty symbols correspond to non-unity and unity Lewis number simulations, respectively. Dashed lines represent power law fits (see text). 


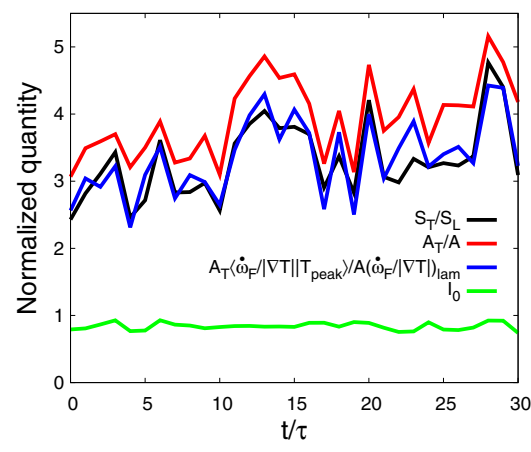

(a) $\mathrm{C}_{\mathrm{ACH} 3}$

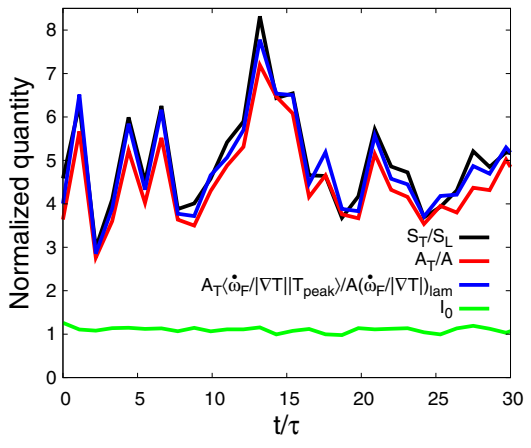

(b) $\mathrm{C}_{\mathrm{ACH} 3,1}$

Fig. 5. Normalized turbulent flame speed $\left(S_{T} / S_{L}\right)$, surface area $\left(A_{T} / A\right)$, Eq. 19, and burning efficiency factor $\left(I_{0}\right)$ plotted against time for cases $\mathrm{C}_{\mathrm{ACH} 3}$. Quantities are shown for every snapshot used for the statistics ( $\sim \tau$ apart). 


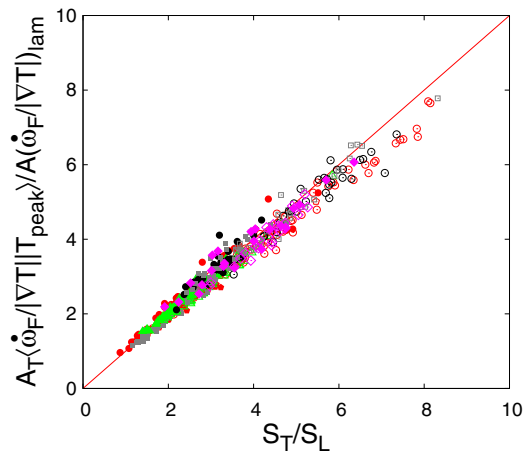

Fig. 6. Scatter plot of the instantaneous $A_{T}\left\langle\dot{\omega}_{F} /|\nabla T| \mid T_{\text {peak }}\right\rangle /\left(A \dot{\omega}_{F, \text { lam }} /\left|\nabla T_{\text {lam }}\right|\right)$ plotted against the instantaneous normalized turbulent flame speed for each snapshot used for all simulations. Filled and empty symbols correspond to non-unity and unity Lewis number simulations, respectively. The colors refer to different fuels and chemistry models (see Fig. 4). 


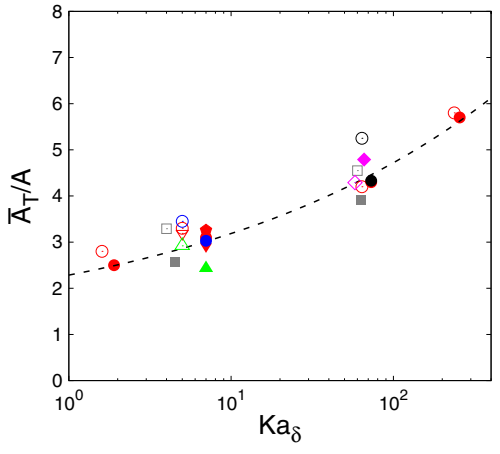

(a) Surface area

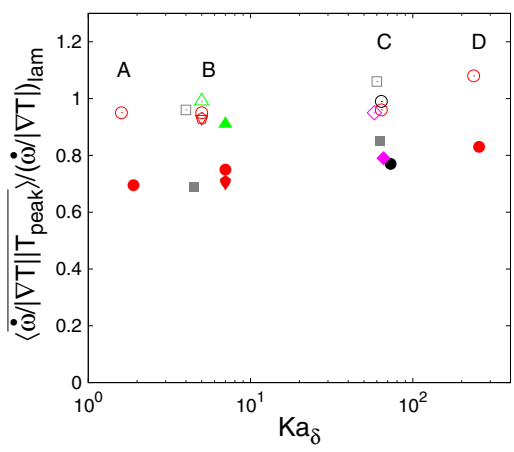

(b) Fuel consumption rate

Fig. 7. Mean turbulent flame surface areas and normalized fuel consumption rates at the corresponding temperature of peak source term. Filled and empty symbols correspond to non-unity and unity Lewis number simulations, respectively. Filled and empty symbols correspond to non-unity and unity Lewis number simulations, respectively. The colors refer to different fuels and chemistry models (see Fig. 4). 


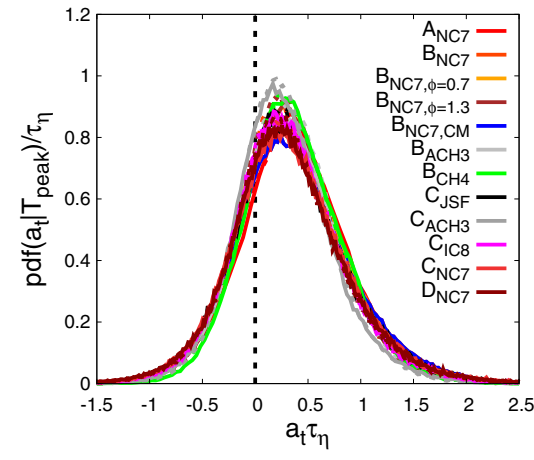

(a) strain rate

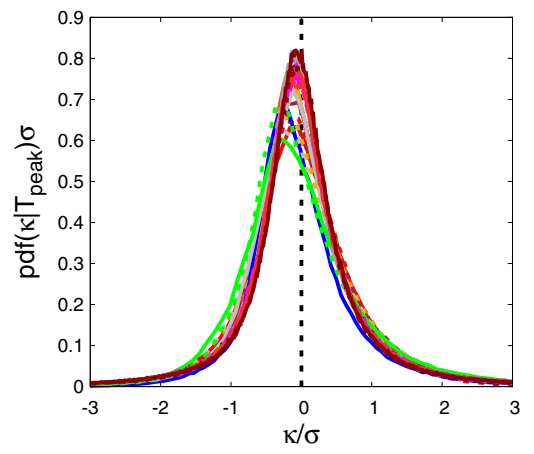

(b) curvature

Fig. 8. Probability density functions of the strain rate and curvature taken on the isosurface of the corresponding temperature of peak source term. The strain rates are normalized by the corresponding Kolmogorov time scales and the curvatures are normalized by their standard deviations. 


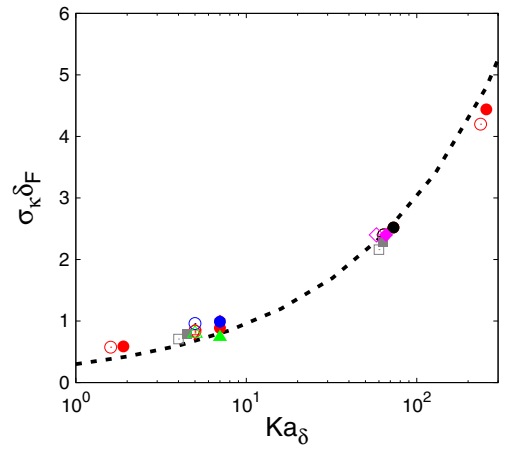

(a) Normalized by reaction zone (b) thickness

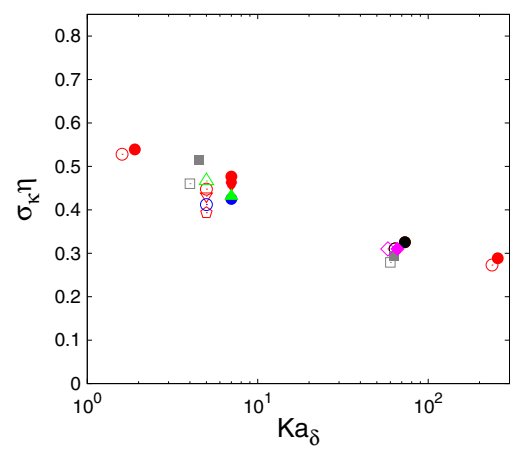

(b) Normalized by Kolmogorov length scale

Fig. 9. Standard deviations of curvature on the reaction zone surface, normalized by the fuel reaction zone thickness and by the Kolmogorov length scale, plotted against the reaction zone Karlovitz number. The dashed line on Fig. 9a represents $\sigma_{\kappa} \delta_{F}=0.3 \sqrt{\mathrm{Ka} \delta}$. Filled and empty symbols correspond to non-unity and unity Lewis number simulations, respectively. The colors refer to different fuels and chemistry models (see Fig. 4). 


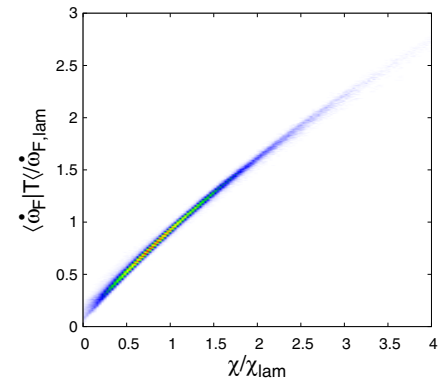

(a) $n-\mathrm{C}_{7} \mathrm{H}_{16}$

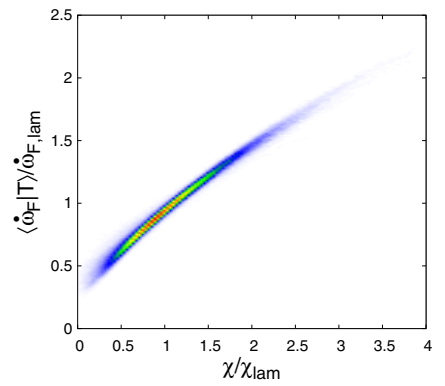

(b) $\mathrm{A}_{1}-\mathrm{CH}_{3}$

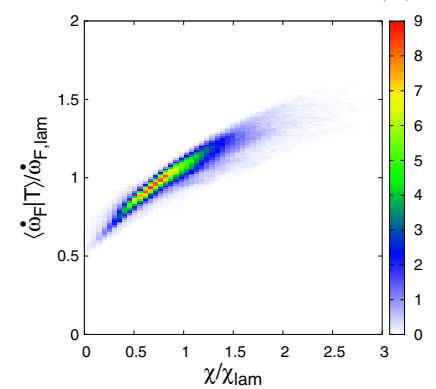

(c) $\mathrm{CH}_{4}$

Fig. 10. Joint probability density functions of the fuel consumption rate and dissipation rate on the isosurface $T=T_{\text {peak }}$ for unity Lewis number cases B. 


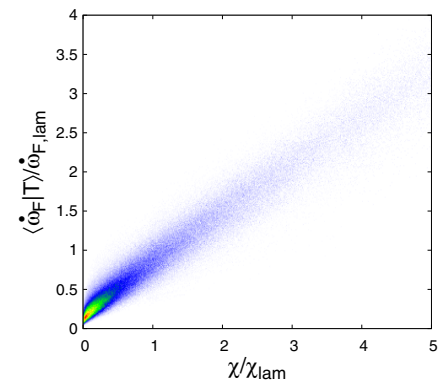

(a) $n-\mathrm{C}_{7} \mathrm{H}_{16}$

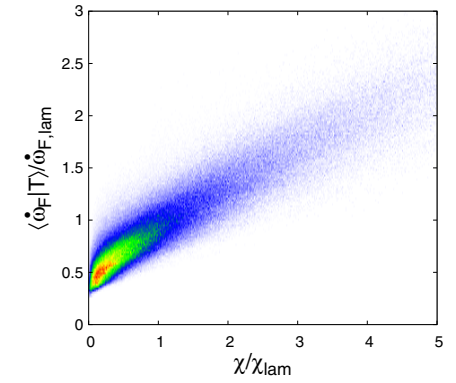

(b) $\mathrm{A}_{1}-\mathrm{CH}_{3}$

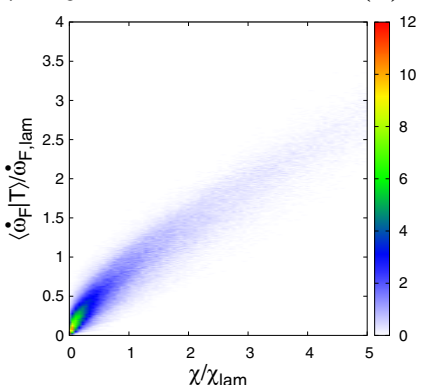

(c) $\mathrm{i}-\mathrm{C}_{8} \mathrm{H}_{18}$

Fig. 11. Joint probability density functions of the fuel consumption rate and dissipation rate on the isosurface $T=T_{\text {peak }}$ for unity Lewis number cases $\mathrm{C}$. 


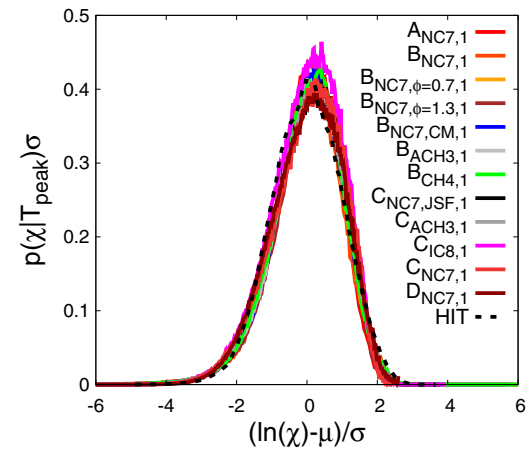

(a) PDF

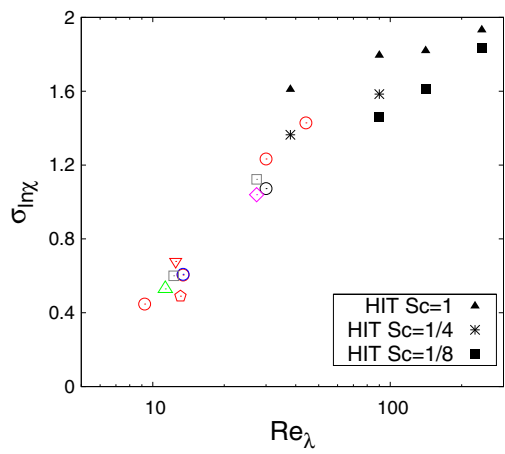

(b) standard deviation

Fig. 12. a) Probability density functions of the dissipation rate taken on the isosurface of the corresponding temperature of peak source term. b) Standard deviations of the PDFs. The dashed line in a) and HIT data in b) correspond to the PDF of dissipation rate of a passive scalar in homogeneous isotropic turbulence from Vedula et al. [54]. Empty symbols correspond to unity Lewis number simulations and the colors refer to different fuels and chemistry models (see Fig. 4). 


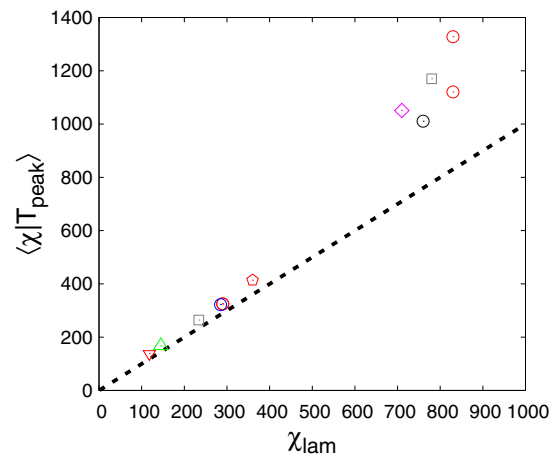

Fig. 13. Means of the probability density functions of $\chi$ at $T=T_{\text {peak }}$ plotted against the corresponding laminar dissipation rate. Empty symbols correspond to unity Lewis number simulations and the colors refer to different fuels and chemistry models (see Fig. 4). 

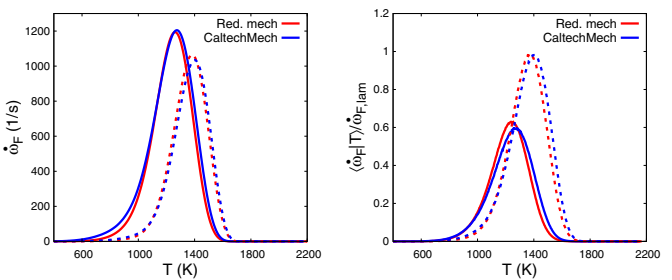

(a) Case laminar
$\mathrm{B}, \quad n-\mathrm{C}_{7},(\mathrm{~b})$ Case $\mathrm{B}, n-\mathrm{C}_{7}$, turbulent
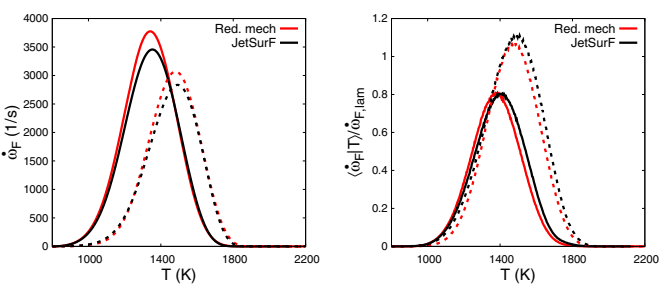

(c) Case $\mathrm{C}, n-\mathrm{C}_{7}$, (d) Case $\mathrm{C}, n-\mathrm{C}_{7}$, turlaminar bulent

Fig. 14. Conditional means of normalized $n$-heptane consumption rate for three different mechanisms. Solid lines correspond to non-unity Lewis number cases while dashed lines correspond to unity Lewis number cases. The fuel consumption rates in the turbulent flames are normalized by the peak value in the corresponding laminar flame. 


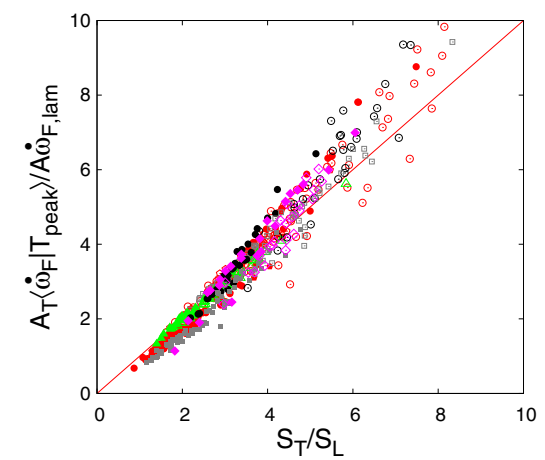

Fig. 15. Scatter plot of the instantaneous $A_{T}\left\langle\dot{\omega}_{F} \mid T_{\text {peak }}\right\rangle /\left(A \dot{\omega}_{F, \text { lam }}\right)$ plotted against the instantaneous normalized turbulent flame speed for each snapshot used for all simulations. Filled and empty symbols correspond to non-unity and unity Lewis number simulations, respectively. The colors refer to different fuels and chemistry models (see Fig. 4). 


\section{List of Tables}

1 Parameters of the simulations previously reported by Lapointe et al. [18]. Subscript 1 denotes simulations with unity Lewis numbers. $\phi$ is the equivalence ratio, $S_{L}$ is the laminar flame speed, $l_{F}=\left(T_{b}-T_{u}\right) /|\nabla T|_{\max }$ is the laminar flame thickness, $l=u^{\prime 3} / \epsilon$ is the integral length scale, $\mathrm{Ka}_{u}$ is the Karlovitz number in the unburnt gas, $\mathrm{Ka}_{\delta}$ is the reaction zone Karlovitz number, $\mathrm{Re}_{t}=u^{\prime} l / \nu$ is the turbulent Reynolds number in the unburnt gas, $\eta_{u}$ is the Kolmogorov length scale in the unburnt gas, $\Delta x$ is the grid spacing, $L$ is the domain width and corresponds to $\sim 5 l$, and $T_{\text {peak }}$ is the temperature of peak fuel consumption rate in the turbulent flame.

2 Parameters of the simulations with different equivalence ratios. See Table 1 for the definitions of the various parameters.

3 Parameters of the simulations with different fuels. See Table 1 for the definitions of the various parameters.

4 Parameters of the simulations with different chemical mechanisms. See Table 1 for the definitions of the various parameters.

5 Correlation coefficient between dissipation rate and fuel consumption rate at $T=T_{\text {peak }}$. Unity Lewis number cases. 


\begin{tabular}{|c|c|c|c|c|c|c|c|c|}
\hline Case & $\mathrm{A}_{n \mathrm{C} 7}$ & $\mathrm{~A}_{n \mathrm{C} 7,1}$ & $\mathrm{~B}_{n \mathrm{C} 7}$ & $\mathrm{~B}_{n \mathrm{C} 7,1}$ & $\mathrm{C}_{n \mathrm{C} 7,1}$ & $\mathrm{C}_{n \mathrm{C} 7,1}$ & $\mathrm{D}_{n \mathrm{C} 7}$ & $\mathrm{D}_{n \mathrm{C} 7,1}$ \\
\hline$T_{u}(\mathrm{~K})$ & \multicolumn{2}{|c|}{298} & \multicolumn{2}{|c|}{298} & \multicolumn{2}{|c|}{800} & \multicolumn{2}{|c|}{800} \\
\hline$\phi$ & \multicolumn{2}{|c|}{0.9} & \multicolumn{2}{|c|}{0.9} & \multicolumn{2}{|c|}{0.9} & \multicolumn{2}{|c|}{0.9} \\
\hline$S_{L}(\mathrm{~m} / \mathrm{s})$ & 0.36 & 0.29 & 0.36 & 0.29 & 2.3 & 1.71 & 2.3 & 1.71 \\
\hline$l_{F}(\mathrm{~mm})$ & 0.39 & 0.43 & 0.39 & 0.43 & 0.25 & 0.27 & 0.25 & 0.27 \\
\hline$u^{\prime} / S_{L}$ & 9.0 & 10 & 18 & 21 & 19 & 25 & 45 & 60 \\
\hline$l / l_{F}$ & 1.1 & 1.0 & 1.1 & 1.0 & 1.2 & 1.1 & 1.2 & 1.1 \\
\hline $\mathrm{Ka}_{u}$ & 78 & 91 & 220 & 280 & 204 & 280 & 740 & 1050 \\
\hline $\mathrm{Ka}_{\delta}$ & 1.9 & 1.6 & 7.0 & 5.0 & 73 & 64 & 256 & 237 \\
\hline $\operatorname{Re}_{t}$ & \multicolumn{2}{|c|}{83} & \multicolumn{2}{|c|}{190} & \multicolumn{2}{|c|}{170} & \multicolumn{2}{|c|}{380} \\
\hline$\eta_{u}(\mathrm{~m})$ & \multicolumn{2}{|c|}{$1.6 \times 10^{-5}$} & \multicolumn{2}{|c|}{$9.0 \times 10^{-6}$} & \multicolumn{2}{|c|}{$7.0 \times 10^{-6}$} & \multicolumn{2}{|c|}{$3.5 \times 10^{-6}$} \\
\hline$\Delta x(\mathrm{~m})$ & \multicolumn{2}{|c|}{$1.8 \times 10^{-5}$} & \multicolumn{2}{|c|}{$1.8 \times 10^{-5}$} & \multicolumn{2}{|c|}{$1.2 \times 10^{-5}$} & \multicolumn{2}{|c|}{$7.0 \times 10^{-6}$} \\
\hline Grid & \multicolumn{2}{|c|}{$11 \times 128^{3}$} & \multicolumn{2}{|c|}{$11 \times 128^{3}$} & \multicolumn{2}{|c|}{$11 \times 128^{3}$} & \multicolumn{2}{|c|}{$11 \times 220^{3}$} \\
\hline$L(\mathrm{~mm})$ & \multicolumn{2}{|c|}{2.30} & \multicolumn{2}{|c|}{2.30} & \multicolumn{2}{|c|}{1.54} & \multicolumn{2}{|c|}{1.54} \\
\hline$T_{\text {peak }}(\mathrm{K})$ & 1240 & 1365 & 1240 & 1365 & 1390 & 1482 & 1440 & 1482 \\
\hline
\end{tabular}

Table 1

Parameters of the simulations previously reported by Lapointe et al. [18]. Subscript 1 denotes simulations with unity Lewis numbers. $\phi$ is the equivalence ratio, $S_{L}$ is the laminar flame speed, $l_{F}=\left(T_{b}-T_{u}\right) /|\nabla T|_{\max }$ is the laminar flame thickness, $l=u^{\prime 3} / \epsilon$ is the integral length scale, $\mathrm{Ka}_{u}$ is the Karlovitz number in the unburnt gas, $\mathrm{Ka}_{\delta}$ is the reaction zone Karlovitz number, $\operatorname{Re}_{t}=u^{\prime} l / \nu$ is the turbulent Reynolds number in the unburnt gas, $\eta_{u}$ is the Kolmogorov length scale in the unburnt gas, $\Delta x$ is the grid spacing, $L$ is the domain width and corresponds to $\sim 5 l$, and $T_{\text {peak }}$ is the temperature of peak fuel consumption rate in the turbulent flame. 


\begin{tabular}{|c|c|c|c|c|}
\hline Case & $\mathrm{B}_{n \mathrm{C} 7, \phi 0.7}$ & $n \mathrm{C} 7, \phi 0.7,1$ & $\mathrm{~B}_{n \mathrm{C} 7, \phi 1.3}$ & $n \mathrm{C} 7, \phi 1.3,1$ \\
\hline$T_{u}(\mathrm{~K})$ & \multicolumn{2}{|c|}{298} & \multicolumn{2}{|c|}{298} \\
\hline$\phi$ & \multicolumn{2}{|c|}{0.7} & \multicolumn{2}{|c|}{1.3} \\
\hline$S_{L}(\mathrm{~m} / \mathrm{s})$ & 0.22 & 0.20 & 0.343 & 0.24 \\
\hline$l_{F}(\mathrm{~mm})$ & 0.52 & 0.53 & 0.37 & 0.47 \\
\hline$u^{\prime} / S_{L}$ & 20.0 & 22 & 16 & 23 \\
\hline$l / l_{F}$ & 1.0 & 1.0 & 1.1 & 0.9 \\
\hline $\mathrm{Ka}_{u}$ & 260 & 280 & 180 & 326 \\
\hline $\mathrm{Ka}_{\delta}$ & 6.3 & 5.1 & 6.7 & 5.3 \\
\hline $\operatorname{Re}_{t}$ & \multicolumn{2}{|c|}{166} & \multicolumn{2}{|c|}{166} \\
\hline$\eta_{u}(\mathrm{~m})$ & \multicolumn{2}{|c|}{$1.1 \times 10^{-5}$} & \multicolumn{2}{|c|}{$9.0 \times 10^{-6}$} \\
\hline$\Delta x(\mathrm{~m})$ & \multicolumn{2}{|c|}{$2.1 \times 10^{-5}$} & \multicolumn{2}{|c|}{$1.8 \times 10^{-5}$} \\
\hline Grid & \multicolumn{2}{|c|}{$11 \times 128^{3}$} & \multicolumn{2}{|c|}{$11 \times 128^{3}$} \\
\hline$L(\mathrm{~mm})$ & \multicolumn{2}{|c|}{2.69} & \multicolumn{2}{|c|}{2.30} \\
\hline$T_{\text {peak }}(\mathrm{K})$ & 1213 & 1308 & 1332 & 1472 \\
\hline
\end{tabular}

Table 2

Parameters of the simulations with different equivalence ratios. See Table 1 for the definitions of the various parameters. 


\begin{tabular}{|c|c|c|c|c|c|c|c|c|}
\hline Case & $\mathrm{B}_{\mathrm{CH} 4}$ & $\mathrm{~B}_{\mathrm{CH} 4,1}$ & $\mathrm{~B}_{\mathrm{ACH} 3}$ & $\mathrm{~B}_{\mathrm{ACH} 3,1}$ & $\mathrm{C}_{\mathrm{ACH} 3}$ & $\mathrm{C}_{\mathrm{ACH} 3,1}$ & $\mathrm{C}_{i \mathrm{C} 8}$ & $\mathrm{C}_{i \mathrm{C} 8,1}$ \\
\hline$T_{u}(\mathrm{~K})$ & \multicolumn{2}{|c|}{298} & \multicolumn{2}{|c|}{298} & \multicolumn{2}{|c|}{800} & \multicolumn{2}{|c|}{800} \\
\hline$\phi$ & \multicolumn{2}{|c|}{0.9} & \multicolumn{2}{|c|}{0.9} & \multicolumn{2}{|c|}{0.9} & \multicolumn{2}{|c|}{0.9} \\
\hline$S_{L}(\mathrm{~m} / \mathrm{s})$ & 0.33 & 0.26 & 0.35 & 0.27 & 2.2 & 1.6 & 2.1 & 1.6 \\
\hline$l_{F}(\mathrm{~mm})$ & 0.47 & 0.41 & 0.41 & 0.49 & 0.26 & 0.32 & 0.24 & 0.28 \\
\hline$u^{\prime} / S_{L}$ & 16 & 20 & 17 & 22 & 18 & 25 & 20 & 27 \\
\hline$l / l_{F}$ & 1.1 & 0.9 & 1.1 & 0.9 & 1.1 & 0.9 & 1.2 & 1.0 \\
\hline $\mathrm{Ka}_{u}$ & 190 & 270 & 205 & 324 & 200 & 331 & 210 & 324 \\
\hline $\mathrm{Ka}_{\delta}$ & 6.8 & 5.4 & 3.5 & 3.1 & 63 & 60 & 66 & 58 \\
\hline $\operatorname{Re}_{t}$ & \multicolumn{2}{|c|}{160} & \multicolumn{2}{|c|}{175} & \multicolumn{2}{|c|}{160} & \multicolumn{2}{|c|}{156} \\
\hline$\eta_{u}(\mathrm{~m})$ & \multicolumn{2}{|c|}{$9.0 \times 10^{-6}$} & \multicolumn{2}{|c|}{$9.1 \times 10^{-6}$} & \multicolumn{2}{|c|}{$6.8 \times 10^{-6}$} & \multicolumn{2}{|c|}{$6.8 \times 10^{-6}$} \\
\hline$\Delta x(\mathrm{~m})$ & \multicolumn{2}{|c|}{$1.8 \times 10^{-5}$} & \multicolumn{2}{|c|}{$1.8 \times 10^{-5}$} & \multicolumn{2}{|c|}{$1.2 \times 10^{-5}$} & \multicolumn{2}{|c|}{$1.2 \times 10^{-5}$} \\
\hline Grid & \multicolumn{2}{|c|}{$11 \times 128^{3}$} & \multicolumn{2}{|c|}{$11 \times 128^{3}$} & \multicolumn{2}{|c|}{$11 \times 128^{3}$} & \multicolumn{2}{|c|}{$11 \times 128^{3}$} \\
\hline$L(\mathrm{~mm})$ & \multicolumn{2}{|c|}{2.30} & \multicolumn{2}{|c|}{2.30} & \multicolumn{2}{|c|}{1.54} & \multicolumn{2}{|c|}{1.54} \\
\hline$T_{\text {peak }}(\mathrm{K})$ & 1622 & 1649 & 1420 & 1527 & 1600 & 1680 & 1447 & 1510 \\
\hline
\end{tabular}

Table 3

Parameters of the simulations with different fuels. See Table 1 for the definitions of the various parameters. 


\begin{tabular}{|c|c|c|c|c|}
\hline Case & $\mathrm{B}_{n \mathrm{C} 7, \mathrm{CM}}$ & $\mathrm{B}_{n \mathrm{C} 7, \mathrm{CM}, 1}$ & $\mathrm{C}_{n \mathrm{C} 7, \mathrm{JSF}}$ & $\mathrm{C}_{n \mathrm{C} 7, \mathrm{JSF}, 1}$ \\
\hline$T_{u}(\mathrm{~K})$ & \multicolumn{2}{|c|}{298} & \multicolumn{2}{|c|}{800} \\
\hline$\phi$ & \multicolumn{2}{|c|}{0.9} & \multicolumn{2}{|c|}{0.9} \\
\hline$S_{L}(\mathrm{~m} / \mathrm{s})$ & 0.36 & 0.29 & 2.3 & 1.71 \\
\hline$l_{F}(\mathrm{~mm})$ & 0.39 & 0.43 & 0.25 & 0.27 \\
\hline$u^{\prime} / S_{L}$ & 18 & 21 & 19 & 25 \\
\hline$l / l_{F}$ & 1.1 & 1.0 & 1.2 & 1.1 \\
\hline $\mathrm{Ka}_{u}$ & 220 & 280 & 204 & 280 \\
\hline $\mathrm{Ka}_{\delta}$ & 7.0 & 5.0 & 73 & 64 \\
\hline $\mathrm{Re}_{t}$ & \multicolumn{2}{|c|}{190} & \multicolumn{2}{|c|}{170} \\
\hline$\eta_{u}(\mathrm{~m})$ & \multicolumn{2}{|c|}{$9.0 \times 10^{-6}$} & \multicolumn{2}{|c|}{$7.0 \times 10^{-6}$} \\
\hline$\Delta x(\mathrm{~m})$ & \multicolumn{2}{|c|}{$1.8 \times 10^{-5}$} & \multicolumn{2}{|c|}{$1.2 \times 10^{-5}$} \\
\hline Grid & \multicolumn{2}{|c|}{$11 \times 128^{3}$} & \multicolumn{2}{|c|}{$11 \times 128^{3}$} \\
\hline$L(\mathrm{~mm})$ & \multicolumn{2}{|c|}{2.30} & \multicolumn{2}{|c|}{1.54} \\
\hline$T_{\text {peak }}$ & 1250 & 1370 & 1400 & 1490 \\
\hline
\end{tabular}

Table 4

Parameters of the simulations with different chemical mechanisms. See Table 1 for the definitions of the various parameters. 
Table 5

\begin{tabular}{c|c|c|c|c|c|c|}
\multirow{2}{*}{ Case } & \multicolumn{5}{|c|}{$r$} \\
\cline { 2 - 7 } & NC7 & $\mathrm{NC}_{\phi=0.7}$ & $\mathrm{NC}_{\phi=1.3}$ & $\mathrm{ACH} 7$ & $\mathrm{CH} 4$ & $\mathrm{iC} 8$ \\
\hline $\mathrm{A}$ & 0.997 & - & - & - & - & - \\
$\mathrm{B}$ & 0.994 & 0.993 & 0.994 & 0.989 & 0.925 & - \\
$\mathrm{C}$ & 0.975 & - & - & 0.934 & - & 0.962 \\
$\mathrm{D}$ & 0.884 & - & - & - & - & - \\
\hline
\end{tabular}

Correlation coefficient between dissipation rate and fuel consumption rate at $T=T_{\text {peak }}$. Unity Lewis number cases. 